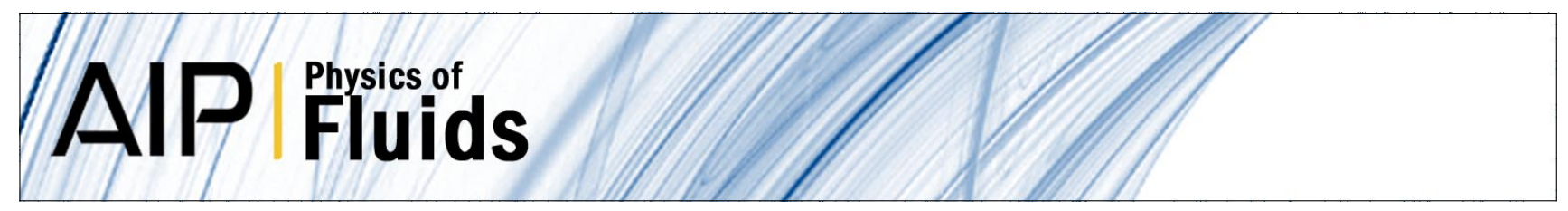

\title{
Experimental investigation of relaminarizing and transitional channel flows
}

Daisuke Seki and Masaharu Matsubara

Citation: Phys. Fluids 24, 124102 (2012); doi: 10.1063/1.4772065

View online: http://dx.doi.org/10.1063/1.4772065

View Table of Contents: http://pof.aip.org/resource/1/PHFLE6/v24/i12

Published by the AIP Publishing LLC.

\section{Additional information on Phys. Fluids}

Journal Homepage: http://pof.aip.org/

Journal Information: http://pof.aip.org/about/about_the_journal

Top downloads: http://pof.aip.org/features/most_downloaded

Information for Authors: http://pof.aip.org/authors

\section{ADVERTISEMENT}

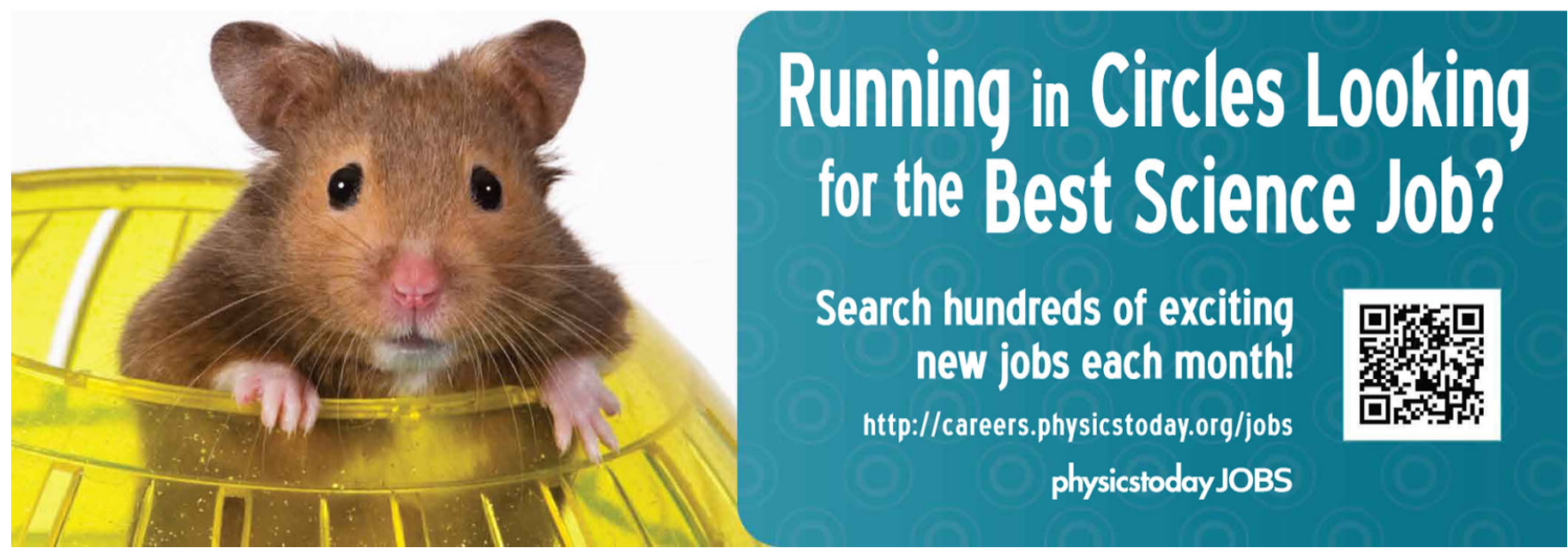




\title{
Experimental investigation of relaminarizing and transitional channel flows
}

\author{
Daisuke Seki ${ }^{1, a)}$ and Masaharu Matsubara ${ }^{2, b}$ \\ ${ }^{1}$ Department of Mathematics and System Development Engineering, Shinshu University, \\ 4-17-1, Wakasato, Nagano 380-8553, Japan \\ ${ }^{2}$ Department of Mechanical Systems Engineering, Shinshu University, 4-17-1, Wakasato, \\ Nagano 380-8553, Japan
}

(Received 8 August 2012; accepted 25 October 2012; published online 20 December 2012)

\begin{abstract}
A hot-wire measurement was conducted in a planar channel flow that originated from a strongly disturbed flow in an entrance channel followed by an expansion channel used to reduce the Reynolds number $(R e)$. From ceasing decrease of the streamwise velocity fluctuation energy and the linear extrapolation of the intermittency factor, the lower marginal $R e$, which is defined as the minimum $R e$ for partial existence of sustainable turbulence, is estimated around 1400 based on the channel width and the bulk velocity. The upper marginal $R e$ at which the intermittency factor reaches one is about 2600 . The flow fields passing a turbulent patch were reconstructed with conditional sampling of the streamwise velocity data based on the time of laminarturbulence interfaces and the reconstructed flow fields indicate a large-scale flow structure across laminar and turbulent parts. This large structure makes it possible for some regions to be at higher $R e$ than the average, so that turbulence can partly survive. The moderate-scale disturbances larger than the turbulent one appear in the non-turbulent parts of the transitional flow, and in these cases the non-turbulent velocity profile is almost identical to the turbulent one. The large-scale fluctuation is observed even over $R e=2600$. This leads to the conclusion that a turbulent channel flow close to the upper marginal Re becomes inhomogeneous. (C) 2012 American Institute of Physics. [http://dx.doi.org/10.1063/1.4772065]
\end{abstract}

\section{INTRODUCTION}

Determination of the marginal Reynolds number $(R e)$, at which shear flow can barely sustain a state of turbulence, is one of the classic subjects of fluid dynamics. In such an extreme condition, systems of disturbance preservation would be distinct, and would probably maintain at a much higher $R e$. Hence, knowledge about the ultimate sustaining system would shed light on the physics of turbulent shear flows. According to the linear stability analysis for a laminar channel flow, any infinitesimal amplitude disturbance decays under the critical Re 7696 based on the bulk (mean) velocity and the channel width. ${ }^{1}$ Even below the critical $R e$, however, the flow becomes turbulent if the initial disturbance is sufficiently strong.

One of the primary studies of the subcritical turbulent channel flow was done by Davies and White $^{2}$ with a stiff water channel facility. Though they did not discuss inlet disturbance in their paper, a sidewall of the channel inlet in the test facility they used had a sharp corner that could cause strong initial disturbances. Their precise pressure drop measurement with various flow rates and channel widths indicates the local minima of the skin friction coefficient at $R e=1440$ and reaches the turbulence value at $R e=2400$. The fact that the marginal $R e$ is much lower than the critical $R e$ for an infinitesimal disturbance leads to the conclusion that there exist two states of flow between the

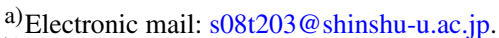

b) Electronic mail: mmatsu@ @hinshu-u.ac.jp.
} 
two $R e$ values. If the initial disturbance is high enough to excite sustainable turbulent disturbances, the flow over the marginal $R e$ becomes turbulent. To determine the marginal $R e$, one must control the initial disturbance to be at a relatively high level.

Patel and $\mathrm{Head}^{3}$ investigated pipe and channel flows around a transitional Re by means of a hot-wire anemometer and a flattened Pitot tube. They introduced sufficient disturbance to induce a transition to turbulence with the sharp corners at the channel entrance and additional wire tripping across the entry. They observed intermittent flow with turbulent bursts in the range of $R e=1380$ to $R e=1800$, while agreement of the skin friction with the $-1 / 6$ power law appears at the much higher $R e=2800$. Badri Narayanan ${ }^{4}$ measured velocity fluctuations in a subcritical channel flow following a fully developed turbulent flow and estimated the marginal (what he termed "critical") $R e=2800 \pm 100$ from extrapolation of the streamwise decay of the fluctuations. Nishioka and Asai ${ }^{5}$ performed a hot-wire measurement in a channel flow strongly contaminated by cylinder-generated vortical disturbances as well as a periodic jet from a wall hole, and they observed a burst appearance at $R e=1330$ or above. Below this $R e$ they observed only low-frequency fluctuation far downstream from the disturbance source. Carlson et al. ${ }^{6}$ investigated natural and artificial turbulent spots in the range of a transitional $R e$ with flake particle flow visualization and found that the spot could be generated by an artificial disturbance at or above $R e$ of about 1330 . They observed strong oblique waves at the sides and the rear of turbulent spots, and spots splitting into two spots downstream, with their gap filled with longitudinal structures. The fully turbulent flow was detected over about $R e=2000$. Alavyoon et $\mathrm{l}^{7}{ }^{7}$ performed visualization of a turbulent spot and they could not generate a turbulent spot below $R e=1470$. They also confirmed the oblique waves and the spot splitting, though the front and rear propagation velocities were higher than estimations by Carlson et al. ${ }^{6}$

These experiments on transitional channel flow indicate that the marginal $R e$ is in the vicinity of 1400, except in the experiment by Badri Narayanan. Numerical studies are consistent with this value, such as that by Orszag and Kells, ${ }^{8}$ who performed direct numerical simulations (DNS) for strongly disturbed channel flow. Though their calculation domain and spectral modes were limited, they confirmed the sustainable non-laminar flow at $R e=1667$, while a channel flow at $R e=667$ showed relaminarization. An another example, a detailed numerical calculation of the transitional channel flow was performed by Iida and Nagano ${ }^{9}$ who revealed that at $R e=1720$ the flow was unsteady in terms of the total skin friction in the whole domain.

Groups including the present authors ${ }^{10,11}$ conducted flow visualization in the transitional channel flow and revealed that the transitional flow was intermittent, with laminar regions, turbulent patches, and large-scale vortices in the non-turbulent regions whose typical streamwise length was 25 channel widths. They also observed laminar flow below $R e=1200$ and turbulent flow uniformly filled with small eddies at $R e=2500$. Fukudome $e t$ al. ${ }^{12}$ recently performed a direct numerical simulation in a large computation box with streamwise and spanwise periodic boundary conditions and found that the patch of turbulence forms a quasi-steady diagonal band. This type of turbulent band was also reported in the direct numerical simulation by Tsukahara $\mathrm{et} \mathrm{al.}{ }^{13}$

In spite of mathematical difficulties, nonlinear analyses of disturbance in the shear flows around the marginal $R e$ were vigorously performed for the last few decades. Recently, a new concept, "edge state" was proposed ${ }^{14-19}$ and successfully described behavior of the nonlinear disturbances in the transitional pipe flow. ${ }^{20-22}$

When considering the marginal $R e$, one can examine the largest vortical scale of turbulent disturbances in the wall unit. Asai and Nishioka, ${ }^{23}$ presenting their experimental results in a transitional boundary layer, pointed out that the spanwise scale of the hairpin vortices was about 50 in the wall unit at the marginal $R e$ and quickly increased to 100 downstream. Alfredsson and Matsubara ${ }^{24}$ proposed that the largest longitudinal vortex should have a spanwise size larger than 50 wall units but should not exceed the geometrical limitation of the flow. This hypothesis well predicted the marginal $R e$ values for several canonical flows.

The main goals of the present study were to determine the marginal $R e$ and to make a quantitative investigation of the transitional channel flow. For these purposes, we built a channel facility that has a fully developed turbulent channel flow and expansion to reduce the $R e$ upstream. The conditional sampling technique for the streamwise velocity measured with a hot-wire anemometer was applied to reconstruct a flow field before and after passing a turbulent patch. The structure of the paper is 
as follows. In Sec. II, we describe the experimental setup and data evaluation. Section III details the streamwise variation of a relaminarizing channel flow and gives consideration to the sufficient channel length. Section IV reports a transitional channel flow focusing on flow statistics at the most downstream position, followed by the conditional sampling results in Sec. V. In Sec. VI, a sustain mechanism of the turbulent patches is proposed, and we present our conclusions.

\section{EXPERIMENTAL SETUP AND DATA EVALUATION TECHNIQUE}

\section{A. Air channel facility}

The experiments were performed in an air channel facility (Figure 1). The air pressurized by a frequency-controlled blower gushes from a perforated pipe positioned in a settling chamber with a cross section of $190 \mathrm{~mm} \times 170 \mathrm{~mm}$. A two-dimensional nozzle with a contraction ratio of 34:1 is followed by channel entrance, expansion and test sections whose wall distance, $d^{*}$, is $5.01 \mathrm{~mm}$. The entrance section is $1000 \mathrm{~mm}$ long and $190 \mathrm{~mm}$ wide. Two tripping wires of $0.5 \mathrm{~mm}$ diameter are strung in the spanwise direction $1 \mathrm{~mm}$ apart from each wall at $100 \mathrm{~mm}$ downstream from the outlet of the nozzle. These wires trigger a transition to turbulence, and the flow becomes a fully developed turbulence flow at the end of the entrance section. In the $1000 \mathrm{~mm}$ long expansion section, the spanwise distance between the channel end walls is symmetrically widened to be $260 \mathrm{~mm}$ at the downstream end, so that the streamwise mean velocity drops to $73 \%$ of the entrance mean velocity in the expansion section. The $2^{\circ}$ tilt of the end wall is low enough to prevent flow separation. The test section following the expansion section is $2800 \mathrm{~mm}$ long, and its downstream end opens to the atmosphere. The idea of the end wall tilting for reducing the $R e$ downstream of a fully developed turbulent channel flow originated from Badri Narayanan. ${ }^{4}$

The coordinates are defined as $x, y$, and $z$, in the streamwise, wall-normal, and spanwise directions, respectively, with the origin at the inlet of the test section on one of the channel walls. All non-dimensional values are based on the wall distance $d^{*}$ and the bulk (mean) channel velocity $U_{b}^{*}$, and the bulk $R e$ of the channel flow is defined as $R e=U_{b}^{*} d^{*} / v^{*}$ with the air kinematic viscosity, $v^{*}$. Dimensional values in the present paper are denoted with a superscript of ()$^{*}$.

\section{B. Measurement technique}

A constant-temperature hot-wire anemometer with a single sensor probe was used for the streamwise velocity measurements. The sensor part of the probe is made of platinum wire $1.5 \mathrm{~mm}$ long and
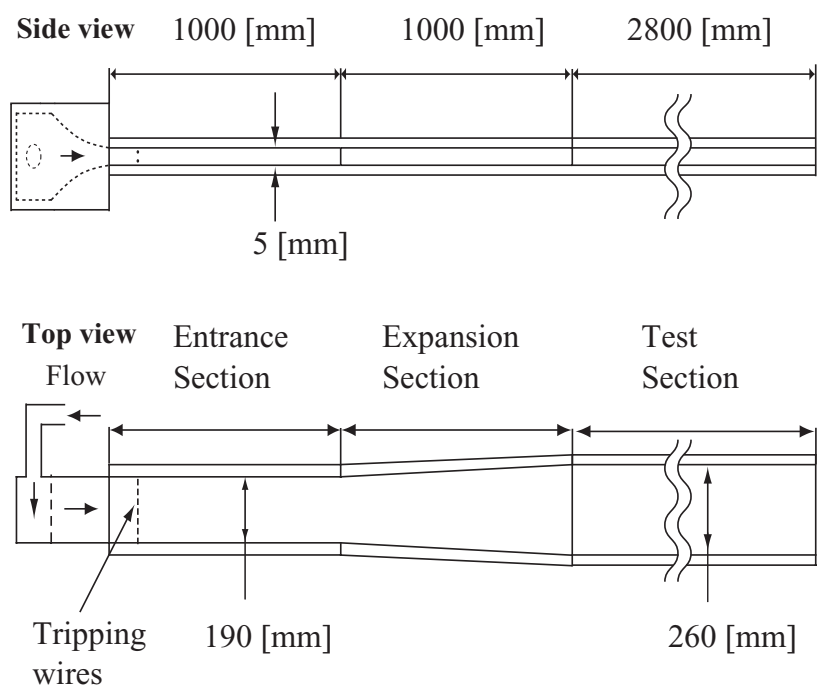

FIG. 1. Air channel flow facility. 
$2.5 \mu \mathrm{m}$ in diameter. Before every set of measurements, velocity calibrations of the anemometer were done in a jet potential core from an axial nozzle connected to the blower for the main channel facility. King's law modified for low velocity ${ }^{25}$ was used for the calibration curve fitting. The temperature variation during the taking of a set of the measurements was monitored to ensure that the temperature drift of the flow from the calibration did not exceed more than $0.7^{\circ} \mathrm{C}$.

The voltages of the hot-wire anemometer were acquired via an analog/digital converter with a sampling frequency of $20 \mathrm{kHz}$, and a sampling time of $30 \mathrm{~s}$ for a streamwise positioning measurement and $300 \mathrm{~s}$ for a detailed measurement at the most downstream position. A wedge mechanism modeled on that used by Klingmann ${ }^{26}$ was employed for probe positioning in the wall-normal direction. It was attached at the ends of two $2300 \mathrm{~mm}$ long bars and was moved by a linear actuator in the streamwise direction. Accuracy of the wall-normal probe movement was checked by the parabolic Poiseuille profile below $R e=1000$. For estimation of the wall position, we employed the symmetric property of the velocity profile for each probe traversing the channel in the streamwise direction.

During each experiment, a hygrometer and a barometer monitored the relative humidity and the atmospheric pressure in the room, respectively. The density and the kinematic viscosity of the flow were calculated from the flow temperature, the relative humidity, and the atmospheric pressure. ${ }^{27}$

Before performing the main experiments, we measured the bulk mean velocity, $U_{b}^{*}$, and the wall distance, $d^{*}$, to assure the accuracy of the bulk Re. The wall distance was measured with an ellipse gauge whose rotation angle was calibrated in advance. The distance ranged from $5.00 \mathrm{~mm}$ to $5.02 \mathrm{~mm}$ with the mean of $5.01 \mathrm{~mm}$ chosen for $d^{*}$.

For $U_{b}^{*}$, we obtained the channel mean velocity, $U_{m}^{*}$, from integration of the time-mean velocity profile $U^{*}\left(y^{*}\right)$ over the channel width using the even tenth-order polynomial function symmetric with respect to the channel center as a fitting function. The accuracy of $U_{m}^{*}$ was not high enough to determine the bulk $R e$ because of hot-wire sensibility to temperature and integration error, so the nozzle pressure drop, $\Delta P^{*}$, was calibrated to $U_{m}^{*}$, and then the relation between the bulk velocity $U_{b}^{*}$ and $\Delta P^{*}$,

$$
U_{b}^{*}=\sqrt{0.3791 \frac{\Delta P^{* 1.112}}{\rho^{*}}}
$$

was obtained, where $\rho^{*}$ is the air density. This $U_{b}^{*}$ was adopted for calculating the bulk $R e$, while the velocity data measured by the hot-wire anemometer were divided by $U_{m}^{*}$ initially, as we expected this would cancel the hot-wire calibration error by the temperature drift.

\section{Procedure for sorting the flow into laminar and turbulent parts}

The intermittency factor, $\gamma$, which is a ratio of total time of turbulence to overall duration, represents a direct index of transitional flows in which there coexist laminar and turbulent parts. In addition, the statistics for each of the laminar and turbulent parts are very valuable for scrutiny of an intermittent flow. The procedure for dividing the flow into laminar and turbulent parts based on the velocity signals has been proposed by Hedley and Keffer, ${ }^{28}$ Kuan and Wang, ${ }^{29}$ and others. Hedley and $\operatorname{Keffer}^{28}$ discussed the difficulties in evaluating the intermittency factor and summarized many of the different detector functions. The greatest difficulty is determination of the thresholds for the detector functions, because they essentially affect the intermittency factor. Kuan and Wang ${ }^{29}$ proposed a further refined technique, the so-called "dual-slope method," which determines the thresholds without ad hoc adjustment.

The first step of the procedure we followed was selecting the detector function that can respond to the turbulence appearance. In our analysis, the sum of a term including the second time derivative and the square of the first time derivative of the streamwise velocity $u$, is proposed as the detector function, as follows:

$$
D(t)=\left(\frac{\partial u}{\partial t}\right)^{2}+\left|(u-U) \frac{\partial^{2} u}{\partial t^{2}}\right|
$$




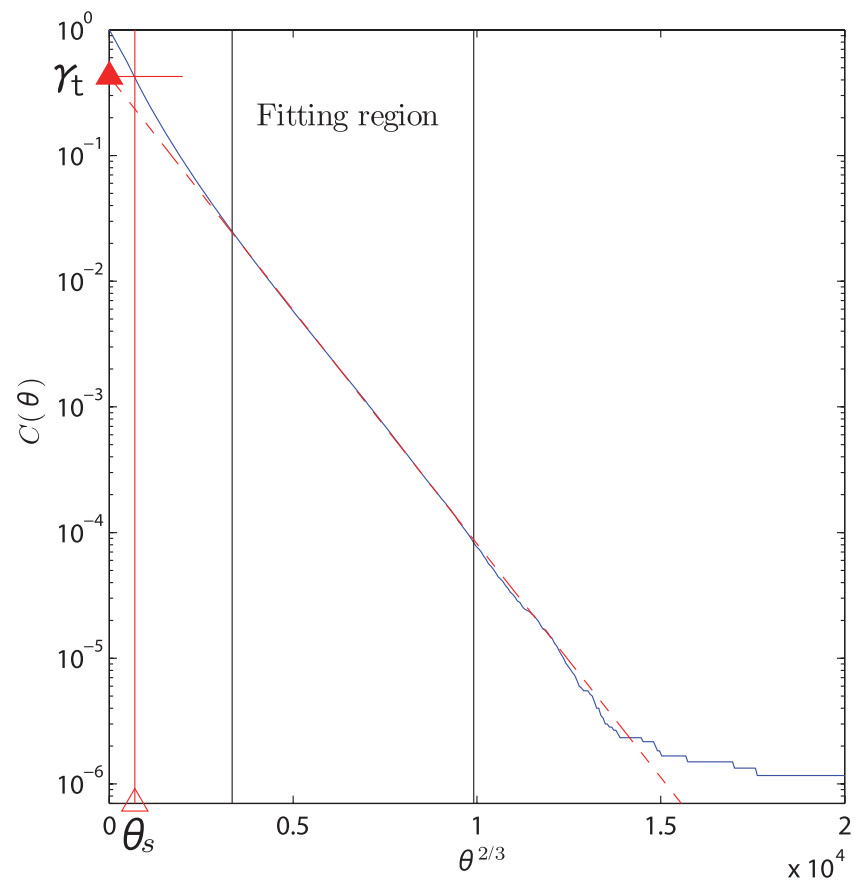

FIG. 2. Single-slope method for the complementary cumulative distribution function $C(\theta)$. The dashed line is the fitting line. Solid and open triangles indicate a tentative intermittency factor $\gamma_{t}$ and a threshold value $\theta_{s}$, respectively.

where $t$ is the time, $U$ is the time mean of $u$, and || denotes the absolute value. The reason for this choice is that the function $D$ is very rarely zero while the velocity signal is highly fluctuated, though each of the terms individually takes zero. In addition, this detector is constant in time if the signal is a pure sine function, and the higher the frequency, the higher the value of the detector. Before calculation of the derivatives, high-frequency noise was removed from the streamwise velocity time signal by a $5 \mathrm{kHz}$ low-pass filter that corresponded to a length scale of about $20 \%$ of the wall distance. We used a fast Fourier transform for the noise filtering and the derivative calculation.

Next, the complementary cumulative distribution function of the detector, $D$, with respect to threshold $\theta$,

$$
C(\theta)=\operatorname{Prob}(D(t) \geq \theta),
$$

was plotted in a log-linear graph. For the dual-slope method, an intersection of two different slopes of the semi-log plotted $C$ was utilized for intermittency factor estimation. However, we found that this method was not functional for the present data, because moderate-scale fluctuation larger than the turbulence scale rounds the corner where the laminar and turbulence slopes meet. Thus, we applied a single-slope method instead of the dual-slope method. This method utilizes the straight slope in high amplitude range of the detector function with a horizontal axis of $\theta^{2 / 3}$ instead of $\theta$, as shown in Figure 2. The ordinate intercept of the slope, shown by a solid triangle, indicated a tentative intermittency factor, $\gamma_{t}$. The applied threshold $\theta_{s}$, shown by an open triangle, was chosen so that the complementary cumulative distribution function at $\theta_{s}$ equals $\gamma_{t}$. The slope was estimated in the range $0.1 \theta_{P}<\theta<0.5 \theta_{P}$, where $\theta_{P}$ is defined as $C\left(\theta_{P}\right)=1 / 600000$. Though we cannot explain why $C$ has a straight slope with $\theta^{2 / 3}$, the slope exists regardless of the wall-normal position and $R e$. Furthermore, we confirmed with human-eye checking of the velocity traces that the ordinate intercept of the slope was appropriate as the intermittency factor.

Applying the threshold $\theta_{s}$ yields the tentative indicator function $I^{\prime}$, that is,

$$
I^{\prime}(t)=\left\{\begin{array}{ll}
1 & \left(D \geqslant \theta_{s}\right) \\
0 & \left(D<\theta_{s}\right)
\end{array} .\right.
$$


Blanks for $I^{\prime}=0$ shorter than three in the non-dimensional time $U_{b}^{*} / d^{*}$ are filled, and then continuous parts for $I^{\prime}=1$ shorter than six are discarded. The conclusive intermittency factor $\gamma$ is obtained as

$$
\gamma=\frac{1}{T} \int_{0}^{T} I d t,
$$

where $I$ is the conclusive indicator function after filling and discarding clusters, and $T$ is the measurement time. In the present paper, the flow state when $I=0$ is nominally called the "laminar part" as the counterpart to the turbulent part, though there sometimes exist strong disturbances in the laminar part.

\section{RELAMINARIZING CHANNEL FLOW}

When the $R e$ of the channel flow in the test section is sufficiently low, turbulent disturbances produced in the entrance section fade out when the flow goes through the expansion and test sections, and the flow becomes laminar if the test channel is long enough. In this section, experimental observations of disturbances with a hot-wire probe traversing a flow in the streamwise direction are reported focusing on the critical point for the sustainable disturbances and the streamwise change of the flow properties.

Streamwise variations of profiles of the mean streamwise velocity, $U$, and distributions of the streamwise velocity fluctuation, $u^{\prime}$, are shown in Figure 3. Before commenting on the flow in the test channel, we will check to see whether the upstream flow at the exit of the entrance section, $x=-200$ is a proper disturbed flow. The bulk $R e$ definition here is based on the velocity in the test section, not the entrance section, so $R e=1390$ and $R e=2680$ correspond to $R e=1900$ and $R e=3670$ in the entrance section, respectively. For $R e=2680$, the mean profile and the fluctuation distribution at $x=-200$ are almost identical to the DNS result ${ }^{30}$ at $R e=5600$, suggesting that the flow at the end of the entrance section is the fully developed turbulent flow. The upstream mean profile at $R e=1390$ is similar to the downstream mean profile at $R e=1940$, though the upstream fluctuation distribution is somewhat lower than the downstream one at $R e=1940$. We consider the difference in the upstream conditions to be the cause of this deviation. The spectra of the velocity fluctuations at both $R e$ values, not shown here, are widely spread in the high-frequency region. In conclusion,
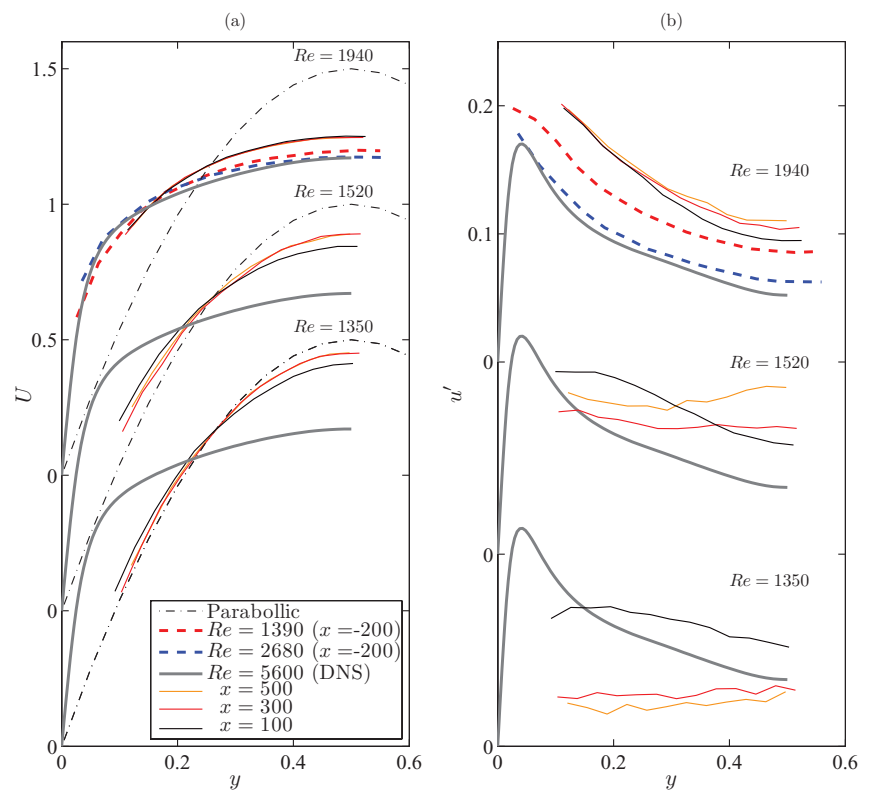

FIG. 3. Streamwise variations of the mean velocity profiles and the distributions of the streamwise velocity fluctuation. (a) Mean velocity profiles. (b) Streamwise velocity fluctuation. 
(a) $R e=1350, y=0.5$

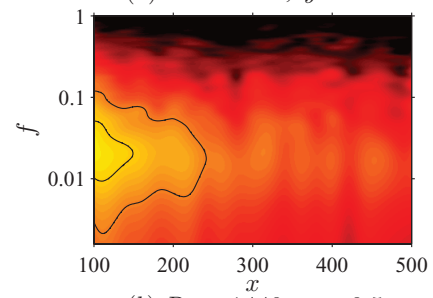

(b) $R e=\stackrel{x}{1440,} y=0.5$

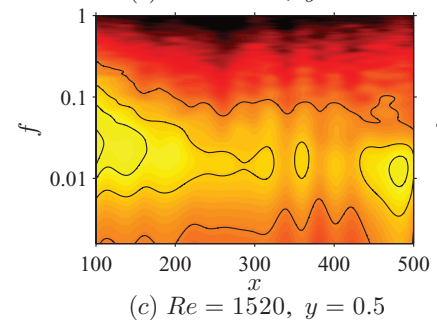

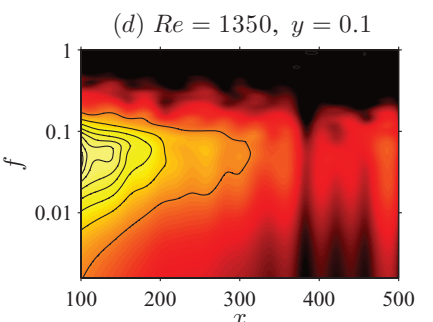

(e) $R e=1440, y=0.1$
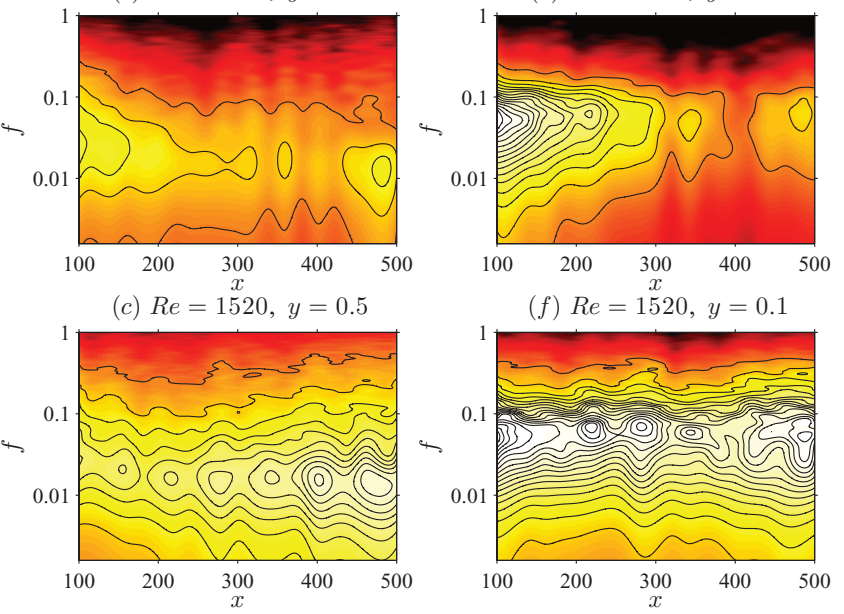

FIG. 4. Streamwise variations of the velocity fluctuation energy spectra.

the velocity fluctuation in the upstream flow has sufficiently high and broad spectral components as the upstream disturbance for the purpose of the present experiment.

The downstream profiles at $R e=1350$ are similar to the laminar parabolic profile. The profiles at $R e=1520$ are almost constant downstream of $x=300$. The profiles remain constant downstream of $x=100$ for $R e=1940$ and they are very similar to a turbulent profile in terms of a flattened profile around the channel center and a high shear near the wall. For $R e=1350$ the wall-normal distributions of the streamwise velocity fluctuation are flat and monotonically decay except for the distribution at $x=100$, which has a weak peak near the wall. The wall peak is more remarkable at $R e=1520$ at $x=100$. The downstream distributions are flat and rises at $x=500$. There are no remarkable change of the fluctuation distributions at $R e=1940$.

The premultiplied power spectra maps shown in Figure 4 reveal the dependence of the flow properties on the streamwise distance. The non-dimensional frequency is defined as $f=f^{*} d^{*} / U_{b}^{*}$. The channel center spectra map at $R e=1350$ has a peak around $f=0.025$ upstream, and it shifts slightly toward a lower frequency downstream with the overall energy going down. At $R e=1440$, the peak value recovers downstream, and then at $R e=1520$ it increases from $x=200$ to $x=400$. The peak frequency at the most downstream position is about 0.013 for $R e \geq 1440$. The equivalent length scale to this peak frequency is $80 d^{*}$ which is much larger than typical scales of the turbulent disturbance. Considering the facts that the low-frequency peak is mainly observed in the transitional flow, and that the scale is equivalent to the turbulent patch interval observed in the flow visualization, ${ }^{6,10,11}$ it appears that this low-frequency peak is the result of turbulent patch passing.

The energy spectra maps near the wall in Figures 4(d)-4(f) have a peak at a high frequency of about 0.05 . Except for the fully turbulent flows, this peak is observed in the maps at $y=0.1$ in the whole measurement cases, i.e., $1180 \leq R e \leq 2360,100 \leq x \leq 500$. According to the flow visualization, ${ }^{10,11}$ this scale is larger than the turbulent disturbance scale, but smaller than that of the turbulent patch passing. The disturbance of this scale will be discussed below. It is notable that the peak value recovering at $R e=1440$ is simultaneous with that in the channel center spectra map. For $R e=1520$, the streamwise variation of the spectra near the wall is roughly constant. 


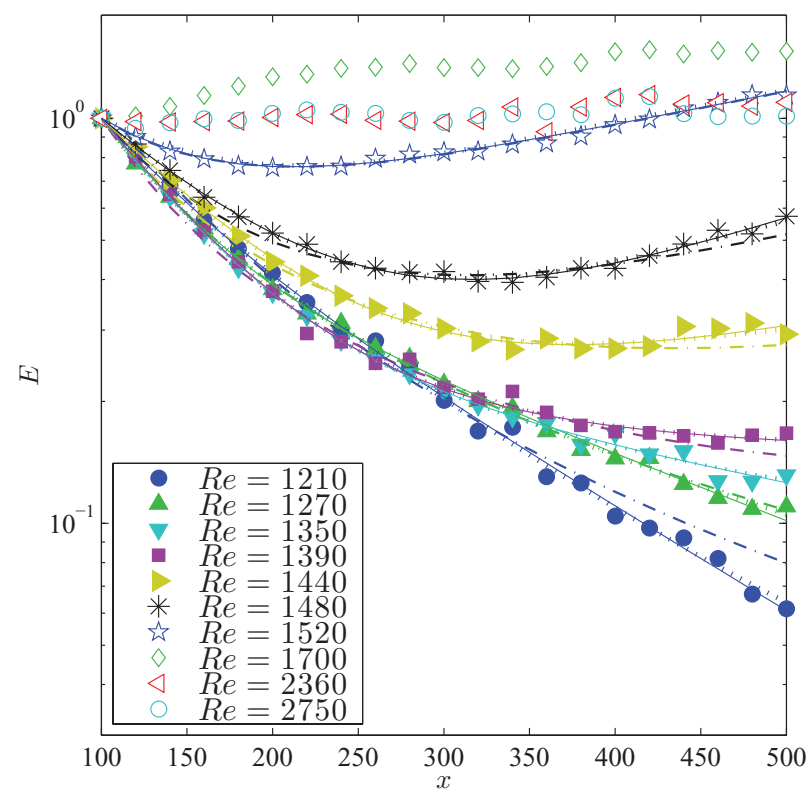

FIG. 5. Streamwise variations of the velocity fluctuation energy. Solid, chain, and dotted lines are fitting curves of Eqs. (6)-(8), respectively.

The streamwise variations of the total energy, $E$, are shown in Figure 5. $E$ was obtained with the trapezoidal numerical integration of the square of the streamwise velocity fluctuation from $y=0.1$ to 0.5 , and normalized with that at $x=100, E_{100}^{*}$. At $R e=1210$, the energy decreases downstream of $x=100$, and the decay rate of energy gets slower downstream. With increasing Re this slowdown is more remarkable, and then at $R e=1440$ the energy starts to rise around $x=400$. Above or at $R e=1700$, the streamwise energy variation keeps almost constant downstream of $x=200$.

The velocity fluctuation energy at $x=500$ is higher than that at $x=100$ for $R e \geq 1520$, so that the flow seems to recover downstream at least superficially. Considering the facts that the energy for $R e \leq 1350$ still decays at the most downstream position, $x=500$, and that the level of the energy there is very low, the flow has a very little chance to recover to a flow state with a sustainable disturbance. We made sure that the profiles of the mean velocity had no remarkable change downstream of $x=300$ in the present $R e$ range, $1210 \leq R e \leq 2660$. For $R e \leq 1350$, the velocity fluctuation downstream of $x=200$ monotonically decreases, keeping its distribution. Therefore, we infer that the test channel length, $x=500$, is sufficient for investigation of the transitional and relaminarizing channel flows, except for very close to the marginal $R e$.

From the streamwise energy variations, we tried to judge if the flow is recovering or not. A simple way is to fit linear lines to the downstream variations in Figure 5, but the inclinations of the lines depend on the streamwise range for the fitting. To avoid this arbitrariness, we tested two-term functions. We chose the simple addition of two exponential terms of $x$ with the slow growth rate $\alpha_{1}$, the rapid growth rate $\alpha_{2}\left(\left|\alpha_{1}\right|<\left|\alpha_{2}\right|\right)$ and their coefficients $A_{1}$ and $A_{2}$ as follows:

$$
E=A_{1} e^{\alpha_{1} x}+A_{2} e^{\alpha_{2} x} .
$$

The addition of two power functions with exponents $\beta_{1}$ and $\beta_{2}\left(\left|\beta_{1}\right|<\left|\beta_{2}\right|\right)$ as

$$
E=B_{1} x^{\beta_{1}}+B_{2} x^{\beta_{2}},
$$

and the combination as

$$
E=C_{1} e^{\alpha x}+C_{2} x^{\beta}
$$



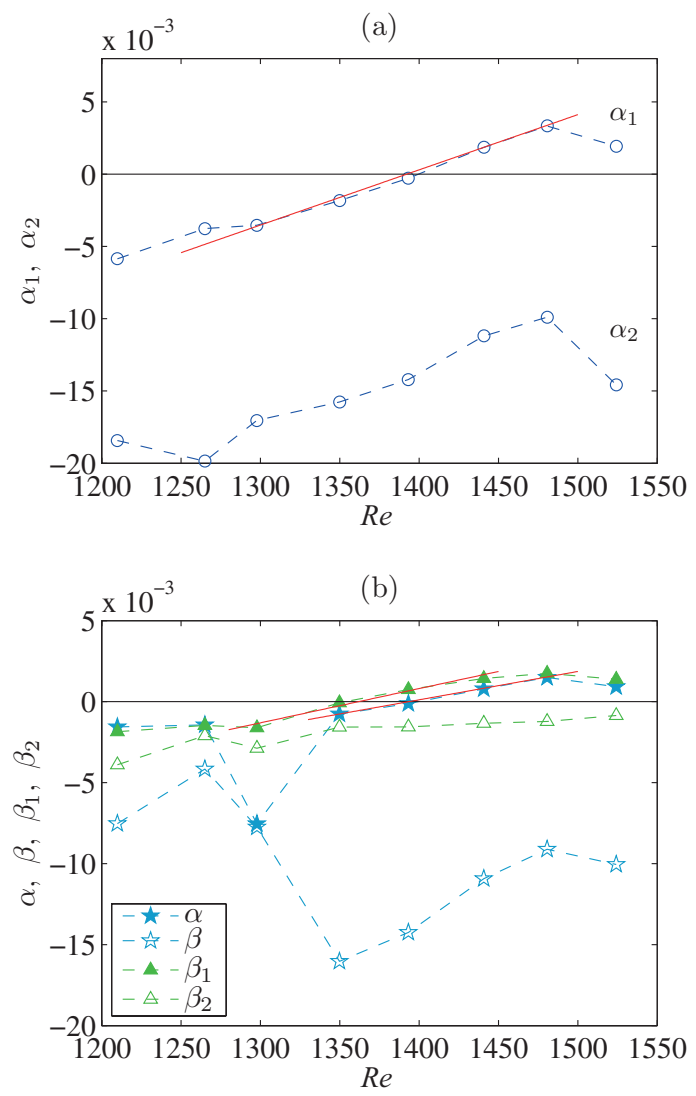

FIG. 6. Re dependence on the growth rates and the exponent for the streamwise energy variation curves. Solid lines are the linear fitting.

were also tested for the fitting. Acceptable fitting for the combination function was achieved with a rapid exponent $\alpha$ so that this $\alpha$ was manually restricted as the rapid growth rate by choosing the best-fitted growth rate for upstream region $x \leq 250$ for the initial value of iterative finding. Figure 5 indicates that the fitting curves of Eqs. (6) and (8) are appropriate for $R e \leq 1520$, where the energy has initial decay. However, the curve Eq. (7) evidently deviates from the measurements. The fittings with the power term for the rapid decay are inappropriate, independent of the formula of the slow term. This is sharp contrast to those with the exponential function. In the other hand, the validity of the formulae for the slow term is not decidable. The fact that the exponential function properly captures the rapid energy decay suggests that the dominant disturbance at the beginning of the energy decay has linear modal properties.

The $R e$ dependence of the exponential coefficients and the exponents are shown in Figure 6. The sign of the slow growth rate, $\alpha_{1}$, switches around $R e=1400$, while the rapid growth rate, $\alpha_{2}$, is below zero in the range of the present estimation. The neutral growth, i.e., $\alpha_{1}=0$, is estimated at $R e=1395$ with the linear fitting of the five measured points near the intercept. This value is one of the criteria for a flow state, laminar or not, from the energy sustainment. The exponent $\beta$ for the mixed function fitting intersects the zero level at $R e=1390$ estimated with the four points around the intersection. The intersection of the slow exponent $\beta_{1}$ around $R e=1350$ is lower than the others. The reason of this shift would be because of the poor fitting with Eq. (7). Badri Narayanan ${ }^{4}$ estimated the marginal $R e$ from the fluctuation energy decay within $10 \leq x \leq 130$. Since the rapid energy decay occurs even when the energy recovers downstream, this streamwise distance is too far upstream for the estimation. This is the reason for his overestimation of the marginal Re. 


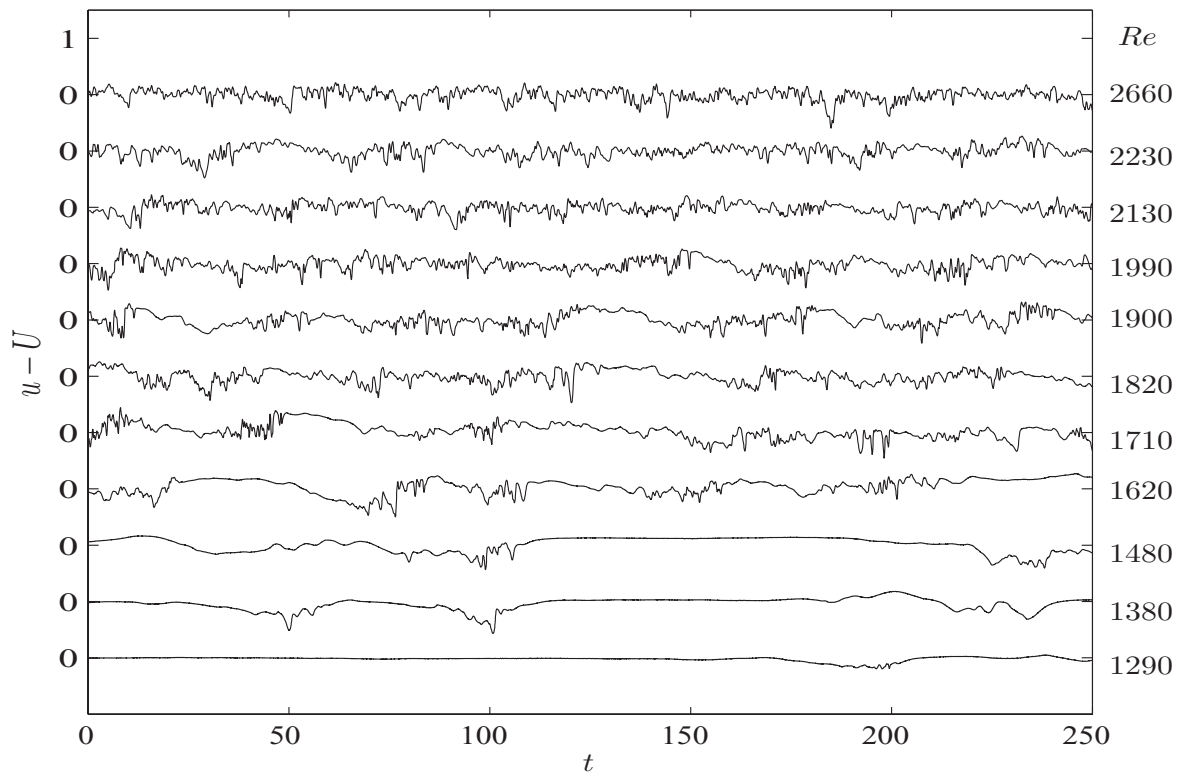

FIG. 7. Time traces of the streamwise velocity at $y=0.5, x=500$.

\section{TRANSITIONAL CHANNEL FLOW}

To obtain more precise flow statistics and conditional averages, we performed concentrated measurements at the most downstream position, $x=500$, with the longer sampling time of $300 \mathrm{~s}$.

Time traces of the streamwise velocity at the center of the channel are shown in Figure 7. The trace at $R e=1290$ indicates a calm state, except for a weak undulation around $t=200$. At $R e=1380$, high-amplitude fluctuations are occasionally observed, but they have no high-frequency components. High-frequency bursts appear in traces at or above $R e=1480$, indicating that turbulent and laminar regions alternately pass through the hot-wire sensor. The total duration of the highfrequency appearance increases with increasing $R e$, and then at $R e=2660$, the laminar duration is hardly observed in the trace. The time traces are characteristic in terms of the acceleration during the turbulent bursts and deceleration of the velocity in the laminar parts, e.g., around $t=70$ at $R e=1620$.

Figure 8 shows the intermittency $\gamma$ and the shape factor $H$ at various wall-normal positions. Since the wall-normal positions are not fixed for each measurement, the $\gamma$ value for a certain position is interpolated with the closest two point values. The $\gamma$ value at the channel center starts to increase at $R e=1380$ and approaches one at $R e=2590$. Near the wall, $\gamma$ also starts to increase at the same $R e=1380$, though its inclination is somewhat lower. It is noticeable that $\gamma$ is roughly constant in the wall-normal direction, except near the wall. As seen in the close-up around $\operatorname{Re}=1500, \gamma$ increases almost linearly. The intersections on the abscissa at each height position are from 1390 to 1410 , estimated with extrapolating linear fits of $\gamma$ in the range $1440 \leq R e \leq 1740$. Below this $R e$, the intermittency factor asymptotically decreases with decreasing $R e$. In the present measurements, the intermittency factor at $R e=1290$ goes down to $0.4 \%$. Taking the finite length of the test channel into account, we concluded that a small amount of turbulent part can survive for a certain distance. From our measurements for the wall-normal positions from $y=0.1$ to 0.5 , the full turbulent flow is realized at $R e=2590$.

Though there is terminological fuzziness for representative $R e$ values concerned with transitional channel flow, in this paper we defined here "marginal $R e$ " as the limit for turbulence sustainment, and we distinguish two situations by using the terms "lower" and "upper" for partial and whole persistence of turbulent flow, respectively. The energy decay and the interpolation of the intermittency factor indicate the lower marginal $R e$ is $1400 \pm 10$. If the upper marginal $R e$ is estimated by the criterion of $99 \%$ intermittency factor, it is approximately 2600 . 


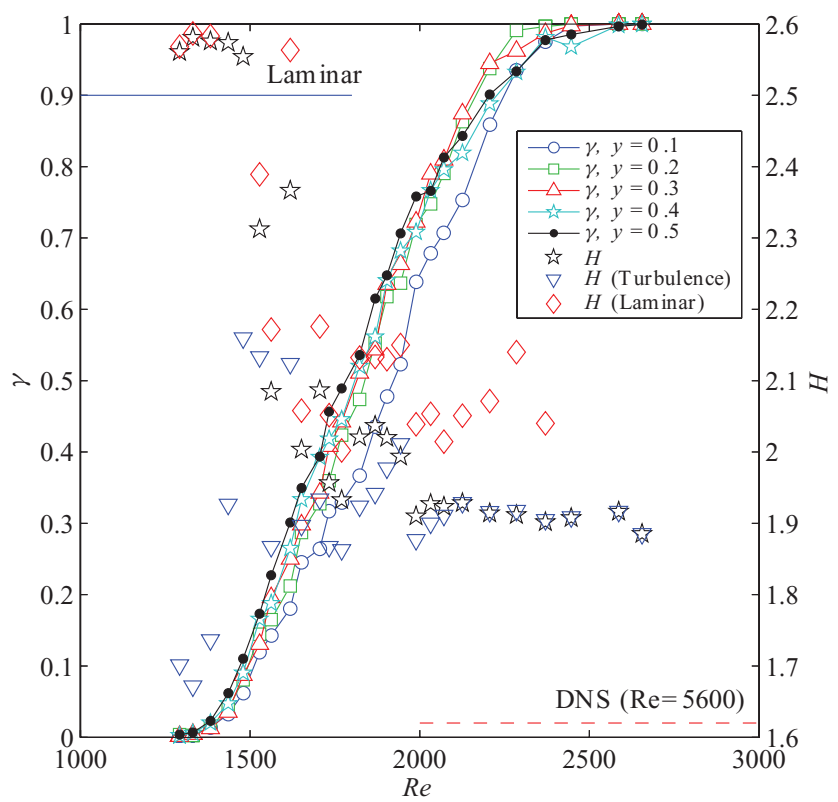

FIG. 8. Intermittency factor $\gamma$ and shape factor $H$ as functions of $R e$. The horizontal solid line is the value of Poiseuille profile, and the dashed line is the turbulent channel at $R e=5600$ (DNS) ${ }^{30}$

The shape factor is defined as follows:

$$
H=\frac{U_{c} \int_{0}^{1}\left(U_{c}-U\right) d y}{\int_{0}^{1} U\left(U_{c}-U\right) d y},
$$

where $U_{c}$ is the center mean velocity. The shape factor rapidly drops from the Poiseuille profile value of 2.5 when the intermittency factor starts to increase and then slightly decreases with increasing $R e$. This variation is in strong contrast to the boundary layer transition due to the free stream disturbance. ${ }^{31}$ The shape factor in the boundary layer case gradually decreases with the intermittency increasing. The shape factor of the laminar part also has a sudden drop at $R e=1500$, while the turbulent part has no remarkable tendency with $R e$ change. The shape factor at $R e=2660$ is still higher than that at $R e=5600 .{ }^{30}$

The number of turbulent patches passing the sensor is counted in the indicator function of the total sampling time and is shown in Figure 9. The value $N$ is the number of the patches during time interval $d^{*} / U_{b}^{*}$. The values from the reconstruction of patch-passing flow fields will be discussed below. In Figure 9(a), just above the lower marginal Re the number at $y=0.5$ sharply increases and reaches the maxima around $R e=1800$, and it then decreases due to merging of the turbulent patches. Close to the wall, the number for $R e<2000$ is lower than that at the center, suggesting that some turbulent patches do not reach the near-wall region.

If the patch count is plotted with the intermittency factor $\gamma$, as in Figure 9(b), the variation at the channel center approximately fits to the parabolic curve, as follows:

$$
N=4 N_{\max } \gamma(1-\gamma) \text {. }
$$

This curve is derived from a simple consideration of the probability. Assuming that trials are independent durations whose state is laminar or turbulent, the number of continuous durations of the turbulent state, $N$, increases with an increase in the number of trials, $n$, as follows:

$$
\frac{d N}{d n}=\gamma(1-\gamma)
$$



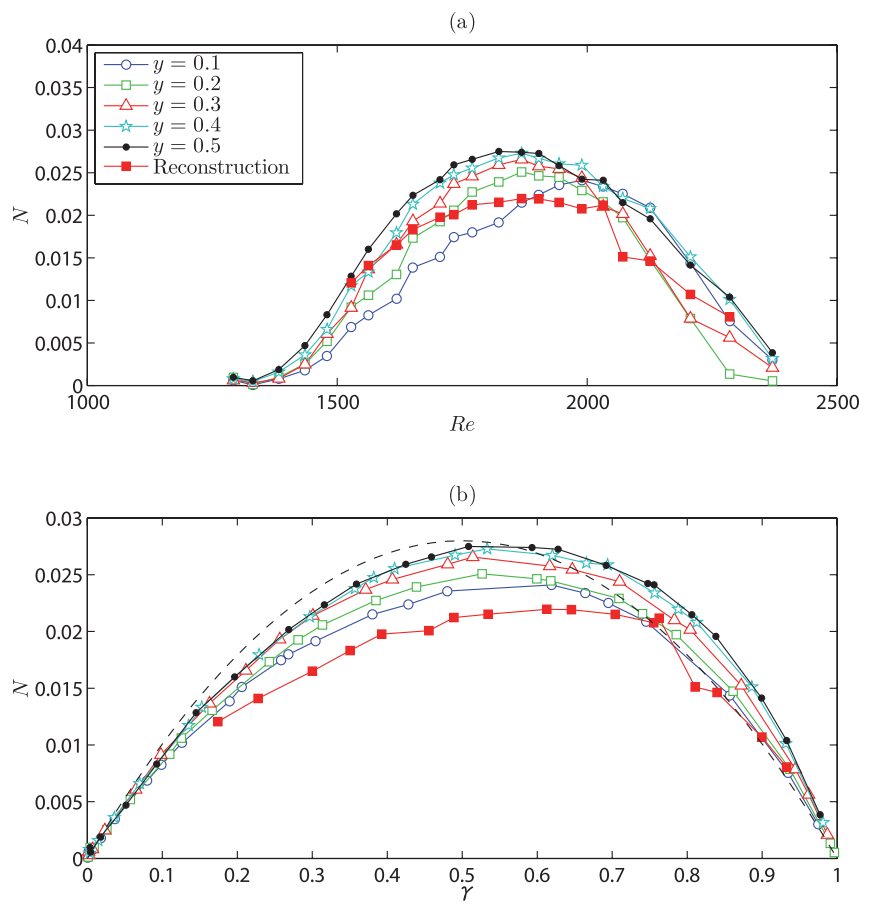

FIG. 9. Number of turbulent patches. (a) $N$ vs. Re. (b) $N$ vs. $\gamma$. The dashed line is the parabolic distribution.

because the intermittency factor $\gamma$ is the probability of turbulence at an additional trial, and $1-\gamma$ is the probability of a laminar state at the trial just before it. This equation is simply integrated with the condition that $N=0$ at $n=0$, and thus,

$$
N=n \gamma(1-\gamma)
$$

The variations of $N$ roughly follow this prediction but the peaks have a slight tendency to shift toward higher $\gamma$. This indicates that the turbulent patches have a tendency to separate more frequently than in the homogeneous probability case.

Profiles of the mean streamwise velocity, $U$, and distributions of the streamwise velocity fluctuation, $u^{\prime}$, at $x=500$ are shown in Figures 10 and 11, respectively. For transitional Re values 1530 and 1950, the velocity fluctuation distributions in the laminar state $(I=0)$ and the turbulent state $(I=1)$ are also plotted. The fluctuations of each state are obtained based on the mean velocity of the state rather than the whole mean velocity. The mean profile at $R e=1260$ agrees with the parabolic laminar profile indicating that the hot-wire measurement at the closest point to the wall is not influenced by additional heat flux to the wall. The fluctuation distributions are less than 5\%, and considering the highly disturbed upstream condition, this fluctuation level is reasonable as a relaminarizing flow. When the $R e$ increases to 1530 , the mean velocity suddenly decelerates in the vicinity of the center, while that near the wall accelerates. The profile drastically changes between $R e=1390$ and $R e=1650$, and the shape factor varies as well. It is noteworthy that the transitional and turbulent mean profiles resemble one another, except close to the lower marginal $R e$. It is also remarkable that there is little difference between the mean profiles for the laminar and turbulent states at $R e=1950$ and this similarity was observed above $R e=1620$. The numerical simulation ${ }^{12}$ at $R e=1660$ also indicates that mean profiles for the laminar and turbulent parts are almost identical.

As shown in Figure 11, the fluctuation distributions up to $R e=1530$ are uniform across most of the channel width. The laminar distribution at $R e=1530$ is also uniform, while an increase near the wall appears in the turbulent distribution. At $R e=1950$ the fluctuation rises near the wall and decreases around the center, so that the distribution becomes very close to that at $R e=2660$. The laminar and turbulent distributions at $R e=1950$ also have a near-wall peak, and the laminar one is 


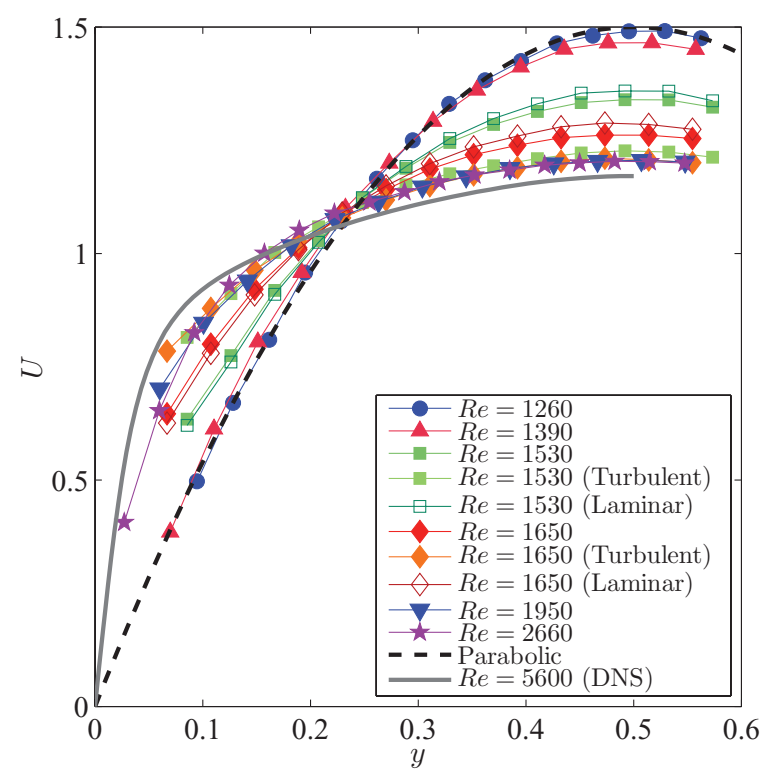

FIG. 10. Mean velocity profiles. The DNS profile is from Kim et al. ${ }^{30}$

slightly lower than the turbulent one. Unexpectedly, the fluctuation at $R e=2660$ is distinctly higher than that at $R e=5600$, suggesting extra disturbance production.

The $R e$ dependence of the velocity fluctuation at $y=0.5$ and $y=0.1$ is shown in Figure 12 . The fluctuation at the channel center is rapidly increasing around $R e=1400$ reaches a peak at $R e$ $=1520$, and then gradually decreases with increasing $R e$. This feature of the rapid increase and the gradual decrease appears in the conditional distributions for the laminar and turbulent cases, though the increase for the turbulent part weakens. The fluctuations for the whole and laminar durations near the wall also show a rapid increase, and the whole duration nearly maintains a constant value, 0.2 for $1800<R e<2500$. The turbulent fluctuation near the wall is relatively high, and its $R e$ dependence is modest. Agreements between the center and near-wall fluctuations for the whole and laminar

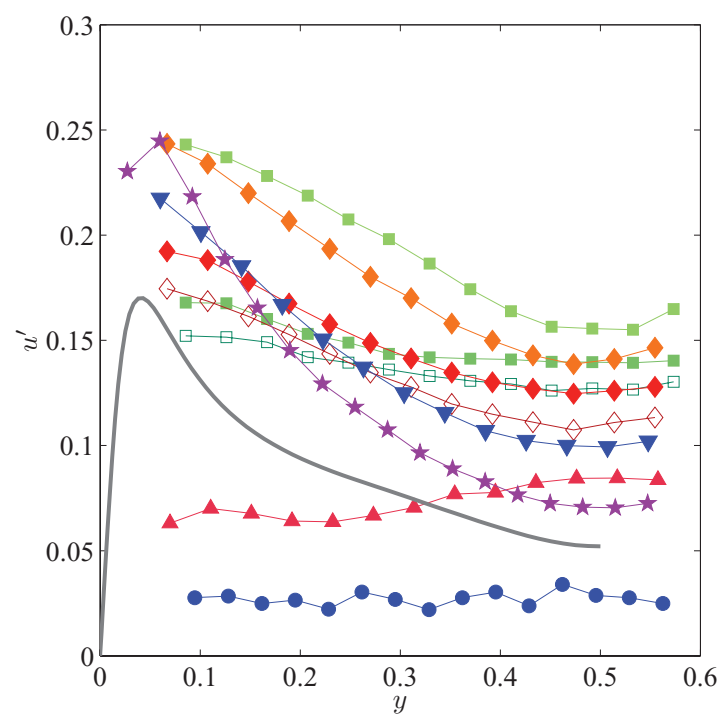

FIG. 11. Velocity fluctuation distributions. The symbols are the same as in Figure 10. 


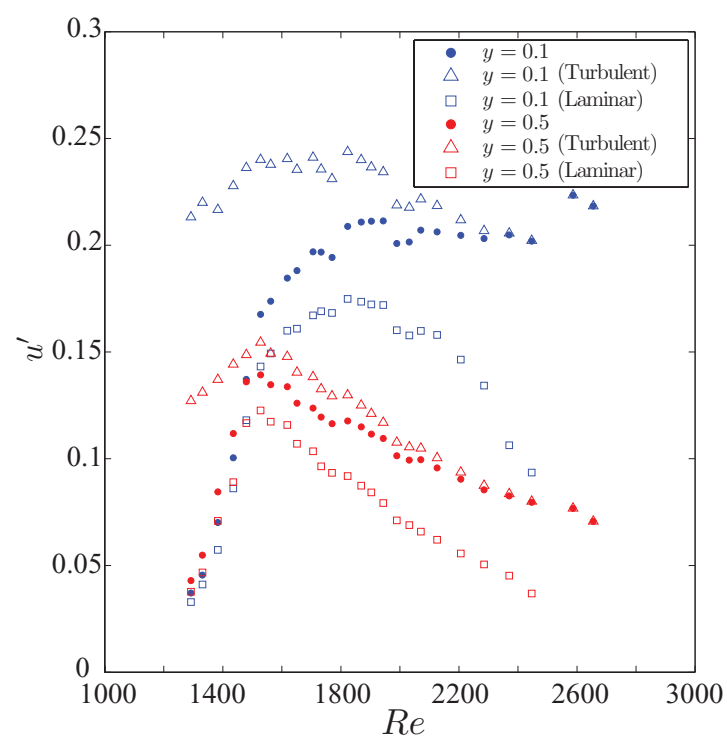

FIG. 12. Re dependence of velocity fluctuations at the center, $y=0.5$, and near the wall, $y=0.1$.

duration in the rapid increase indicate that a uniform distribution of the fluctuation sensitively rises just above the lower marginal $R e$.

At the middle of the transitional $R e$ range, the turbulent-like mean velocity profiles with the low shape factor and high fluctuation are observed, even for the laminar part. This suggests intense wall-normal exchange of the streamwise momentum comparable to the Reynolds stress in a turbulent channel flow. The velocity signal in the laminar part, however, contains no high-frequency disturbance. To seek disturbances transporting the momentum in the laminar part, we surveyed velocity spectra. Figure 13 is a contour map of the premultiplied frequency spectra of the streamwise velocity fluctuation at the channel center. The thick black and gray (blue) lines indicate peak positions of the spectra intensity. We note that a peak at a very low frequency is distributed in a wide range of $R e$ with the maximum around $R e=2100$. In the middle range of the transitional $R e$, these values are very close to the patch appearance, $N$, indicated by the light gray (aqua) line. The length scale corresponding to the peak decreases from $65 d^{*}$ to $25 d^{*}$ with increasing $R e$ and is the same order of magnitude as the patch appearance interval investigated in the flow visualization. ${ }^{10,11}$ It is noteworthy that this large-scale peak at $f=0.04$ is observable even in the full turbulent state at

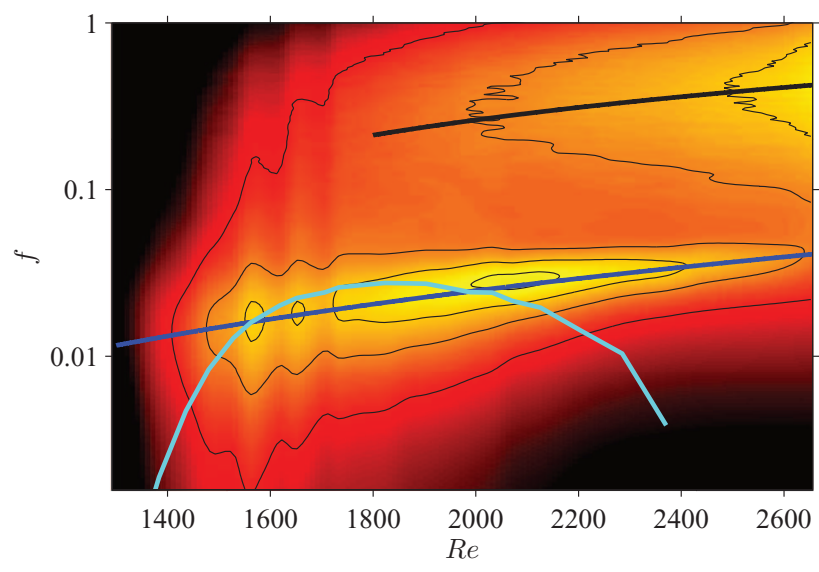

FIG. 13. Contour map of the premultiplied energy spectra at $y=0.5$. 


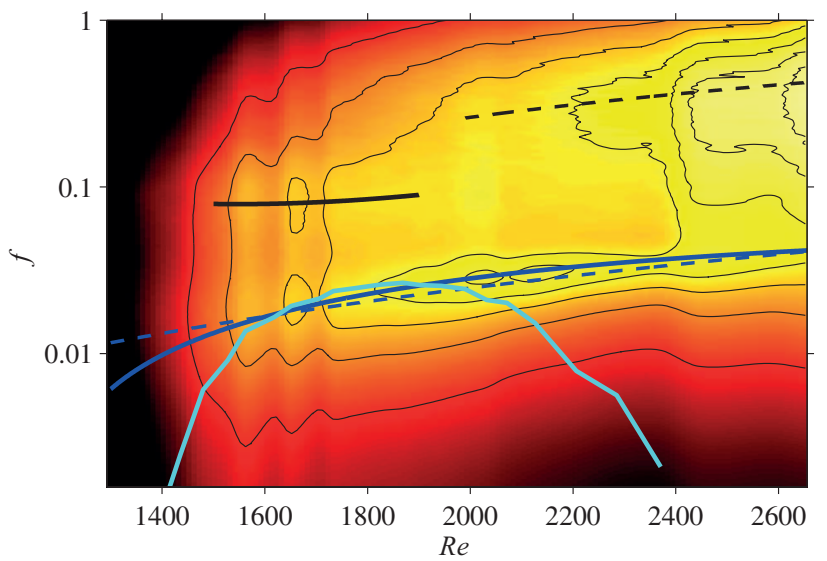

FIG. 14. Contour map of the premultiplied energy spectra at $y=0.3$. Dashed lines indicate the peak positions at $y=0.5$. The contour space is the same as in Figure 13.

$R e=2660$. In the spectral map at $y=0.3$ shown in Figure 14, the high-frequency peak intensifies and the low-frequency peak is still confirmed. A new peak appears between the very low and high peaks in the low-Re region. As seen in the near-wall spectra map of Figure 15, this moderate-frequency peak is more distinct in a whole transitional $R e$ range.

The velocity fluctuation energy distributions over the wall-normal direction and the $R e(R e-y)$ are mapped with frequency filters in Figure 16. The total energy distribution spreads from the near-wall region to the channel center just above the lower marginal $R e$, and it concentrates at the near-wall region with increasing $R e$. The large-scale energy was evaluated by means of low-pass filtering with a cutoff frequency $f_{L}=0.04$. It is evident that around $R e=1500$ there exists a peak at the center. The velocity fluctuation after the band-pass filtering has a peak near the wall in the whole transitional $R e$ range. The lower and higher cutoff frequencies are $f_{L}$ and $f_{H}=0.15$, respectively. The fluctuation distribution with the near-wall peak suggests disturbance generation caused by the wall-normal velocity gradient of the mean shear and the wall-normal velocity fluctuation, $v^{\prime}$, through the term $v^{\prime} \partial U / \partial y$. This moderate-scale disturbance will be discussed again after the proceeding conditional sampling analysis. On the other hand, we inferred that the large-scale fluctuation peak at $R e=1500$ was not caused by vortical disturbance, because the term could not produce high fluctuation at the channel center where $\partial U / \partial y=0$.

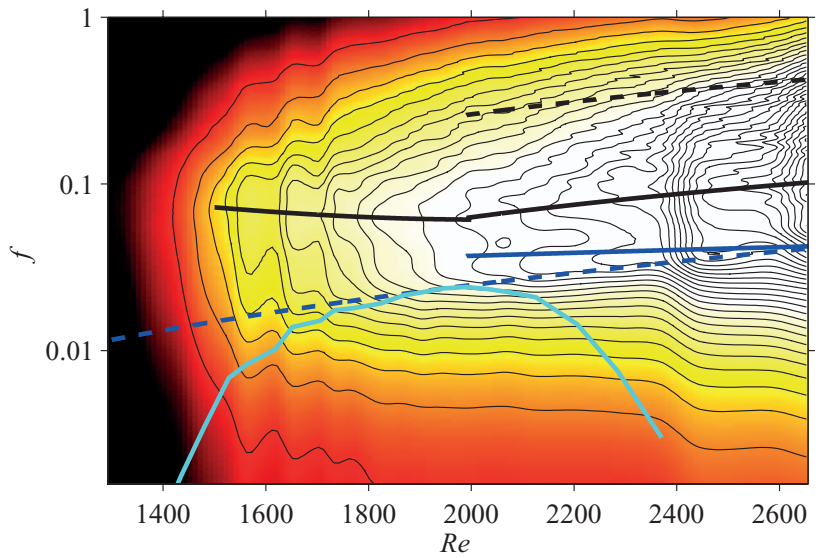

FIG. 15. Contour map of the premultiplied energy spectra at near the wall at $y=0.1$. Dashed lines indicate the peak positions at $y=0.5$. The contour space is the same as in Figure 13 . 
(a) $\mathrm{T}$ o t a 1

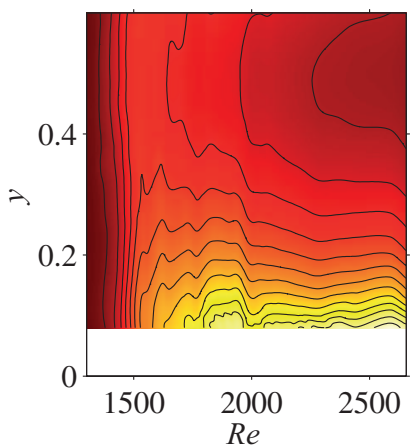

(c) B a n d - p a s s

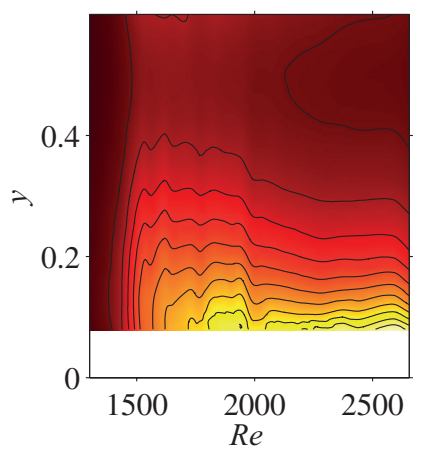

(b) L o w - p a s s

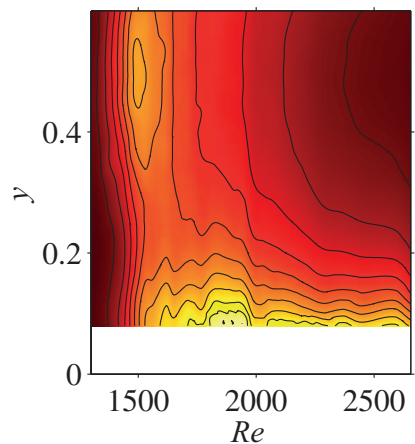

(d) $\mathrm{Hig} \mathrm{h}-\mathrm{p}$ a s s

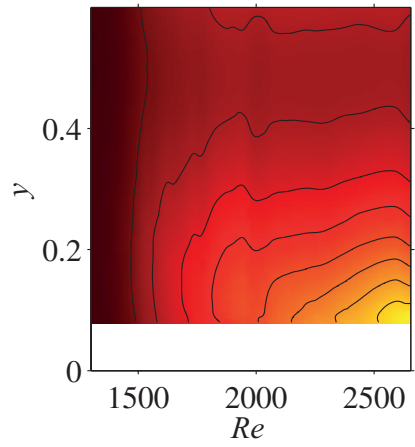

FIG. 16. Distributions of energy of the streamwise velocity fluctuation in the $(R e-y)$ plane. Counter spacing is $0.4 \%$ for (a), $0.2 \%$ for (b)-(d).

The high-frequency energy whose scale corresponds to the turbulent disturbance scale is obtained from the high-pass filtered velocity fluctuation with the cutoff frequency $f_{H}$. Its wall-normal distribution is very similar to the band-pass one in terms of the near-wall peak, while the peak value increases with $R e$, in contrast to the constant peak value in the band-pass map over $R e=1800$. Therefore, we conclude that this high fluctuation is due to the turbulent disturbance inside the patch.

\section{CONDITIONAL SAMPLING ANALYSIS AROUND AN INTERFACE BETWEEN THE LAMINAR AND TURBULENT PARTS}

We performed conditional sampling on the basis of an interface between turbulence and laminar states to investigate the detailed properties of the flows around the interface. The streamwise velocity is conditionally sampled with a reference time at the flow state switching from laminar to turbulent (LT), or from turbulent to laminar (TL). The samples are restricted by the length of the pure laminar or turbulent flow state. We confirmed that this restriction has no significant influence on the conditionally sampled results if it is longer than $10 d^{*} / U_{b}^{*}$, and we thus fixed the minimum length of the pure flow state at $N^{-1}$ of the channel center, which is always much longer than $10 d^{*} / U_{b}^{*}$ (see Figure 13).

Figure 17 shows the time variations of the local (or temporal) Reynolds number, $R e_{s}$, which is calculated by means of integration of the conditionally averaged velocity with the tenth even polynomial fitting the same as the estimation of the bulk mean velocity. The differential time, $\Delta t$, is measured from the reference time at the interface. Rough similarities of variations of $R e_{s}$ around the laminarturbulent interfaces are illustrated when $R e_{s}$ is divided by $R e$. In the variation of LT, the $R e_{s}$ rapidly increases just after the minimum at the interface time $(\Delta t=0)$, up to the local maximum around $\Delta t=1$, and after short reduction it increases again. Just before the interface time, $R e_{s}$ decreases but not as rapidly as the turbulence side, and before the mild variation around $\Delta t=-5$ it constantly 

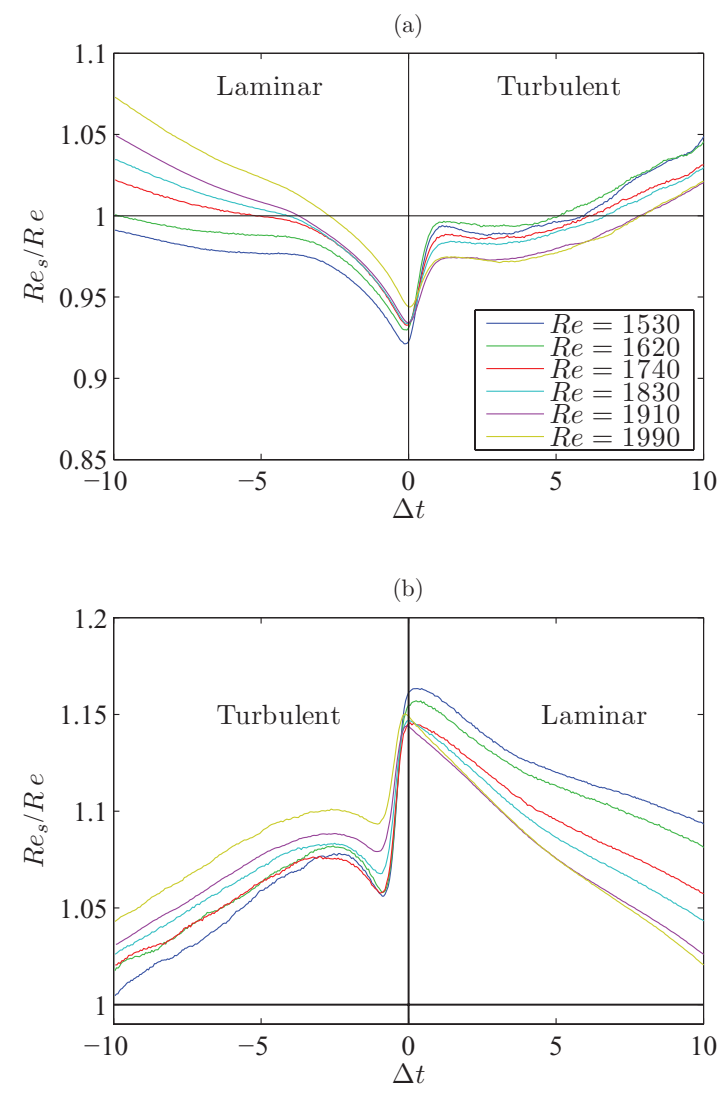

FIG. 17. Conditional sampled time variations of $R e_{s} / R e$ around interfaces between laminar and turbulent parts. (a) From a laminar part to a turbulent part (LT). (b) From a turbulent part to a laminar part (TL).

decreases. The far laminar side slope around $\Delta t=-10$ becomes steeper as the bulk $R e$ increases, and the extrapolated values at the interface time $(\Delta t=0)$ are about $R e_{s} / R e=0.96$ independent of $R e$. On the other hand, the far turbulence side slopes keep a constant inclination, and their extrapolated value at $\Delta t=0$ drops from 0.95 to 0.92 with increasing $R e$. Variations of $R e_{s} / R e$ for the interface TL (Figure 17(b)) have valleys just before they reach peaks at the interface time. There also exist local maxima before the minima following the long slope of $R e_{s}$ on the turbulent side. After the peaks at the interface time, $R e_{s}$ monotonically decreases, with slowing around $\Delta t=4$. The laminar and turbulent slopes apart from the interface have the same dependence on $R e$ as in the LT case. The extrapolated values of the far laminar slopes at the interface time are about $R e_{s} / R e=1.14$ regardless of $R e$ and those of the far turbulent slopes increase from 1.10 to 1.14 with increasing $R e$.

The far slopes of $R e_{s}$ on the laminar side in Figure 17(b) seem to be able to connect smoothly to those in Figure 17(a). This is also true for the far variations on the turbulent sides, and thus the flow from one turbulent patch to the next can be reconstructed by placing the two conditionally sampled averages (LT and TL), side by side, while choosing an appropriate clipping time. For the reconstruction, we minimized the gaps of $R e_{s}$ at the clipping time, keeping time durations on both sides of the interface equal, and we restricted the ratio of sum of the turbulent durations to the whole duration to be equal to the intermittency $\gamma$ at $y=0.5$. The inverse numbers of the optimized intervals for the reconstruction plotted in Figures 9(a) and 9(b) are close to the average number of the turbulent patch passing, though they are slightly smaller and the peak position shifts more to the higher $\gamma$.

Figures 18 and 19 show the reconstructed variations of $R e_{s}$, the ensemble-averaged velocity, $\tilde{U}$, and the velocity fluctuation, $\tilde{u}$, for a duration longer than one period. For $\tilde{U}$ the time mean velocity, $U$, was subtracted. The connections are almost smooth at the clipping time not only in the 


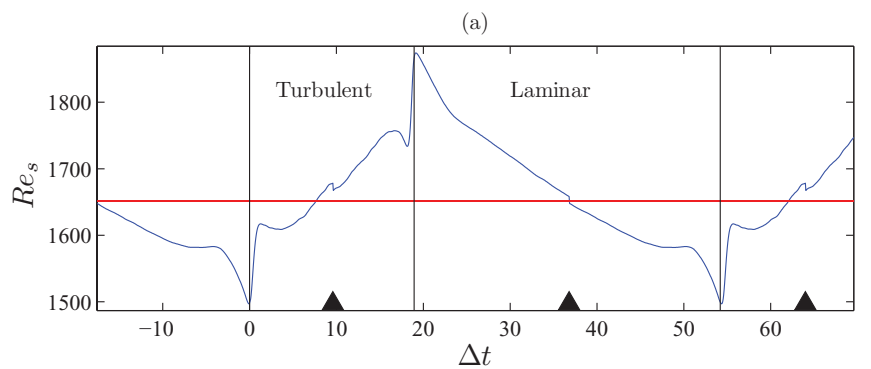

(b)

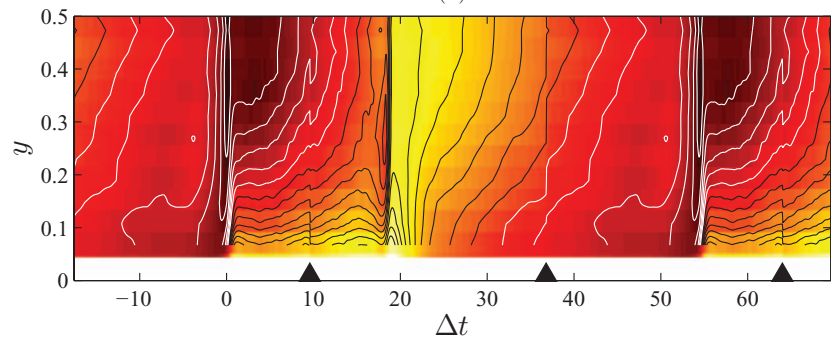

(c)

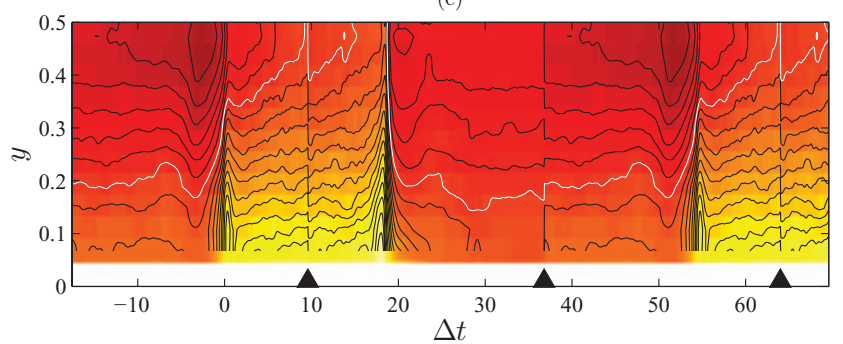

FIG. 18. Space-time distributions of conditionally sampled results at $R e=1650$. (a) $R e_{s}$ : Solid vertical lines and solid triangles represent the interface time and the clipping time, respectively. Solid horizontal line indicates the bulk $R e$. (b) Contour plot of $\tilde{U}-U$. Higher value regions are lightly filled. The contour spacing is $2 \%$, and negative contour lines are white. (c) Contour plot of $\tilde{u}$. The contour spacing is $1 \%$, and white lines are for $\tilde{u}=14 \%$.

$R e_{s}$ variation but also in the averaged velocity and the fluctuation contour maps. This means that the reconstruction well captures the nature of the velocity field with passing a turbulent patch.

Figures 18 clearly indicates that variation of the local $R e_{s}$ is drastic in the whole reconstructed duration. The total amplitude from the peak to the valley of $R e_{s}$ is about $20 \% R e$. The intersections of $R e_{s}$ across the bulk $R e$ are close to the clipping time, and the variation has approximate rotational symmetry with respect to these intersections. This fact is consistent with the similarity of the conditionally averaged laminar and turbulence velocity profiles, in spite of the high amplitude of $R e_{s}$. Considering the wall-normal integration of the continuity equation, we deduced from the drastic variation of the $R e_{s}$ that a large-scale field of the streamwise velocity accompanies that of the spanwise velocity. The direct numerical simulation by Fukudome et al. ${ }^{12}$ shows that large-scale flow in the spanwise-streamwise plane circulates around the center of the inclined turbulent band. The drastic $R e_{s}$ change strongly suggests the large structure similar to the flow with the turbulent band.

The laminar side velocity profile just after the turbulent patch constantly decreases. At the leading edge of the turbulent patch $(\Delta t=0)$, the velocity is low at the channel center, while the laminar side velocity close to the turbulent part trailing edge $(\Delta t=19)$ accelerates especially near the wall. In contrast to the velocity profiles, the fluctuation distribution is almost constant in the laminar part, except for reduction at the channel center before the leading edge. The turbulent part has local intensification near the wall at the leading and trailing edges, and the fluctuation in the middle gradually increases. The highly fluctuated trailing edge is also investigated in a turbulent spot artificially generated in the channel flow. ${ }^{32}$ 


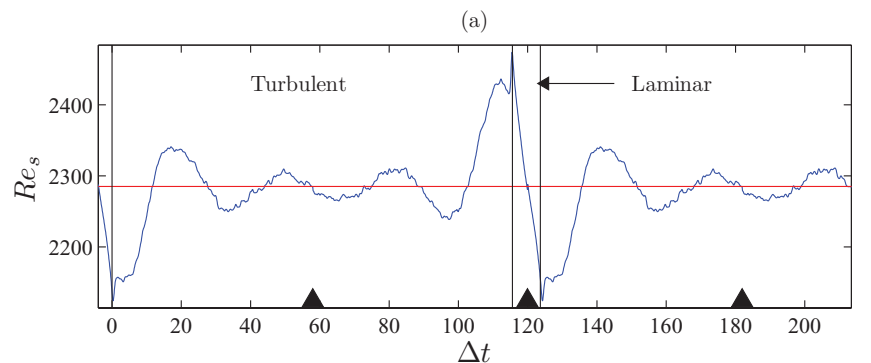

(b)

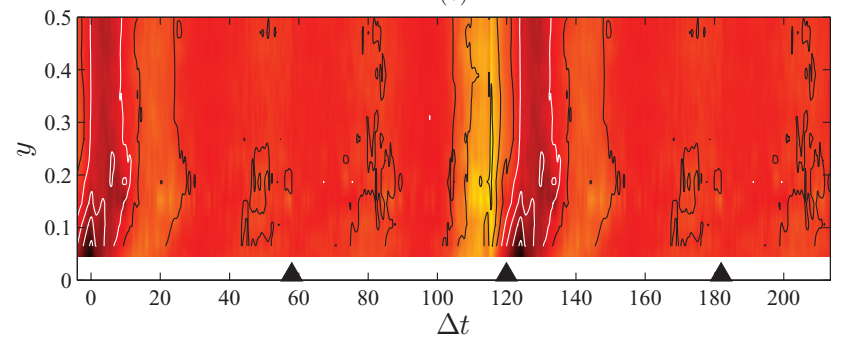

(c)

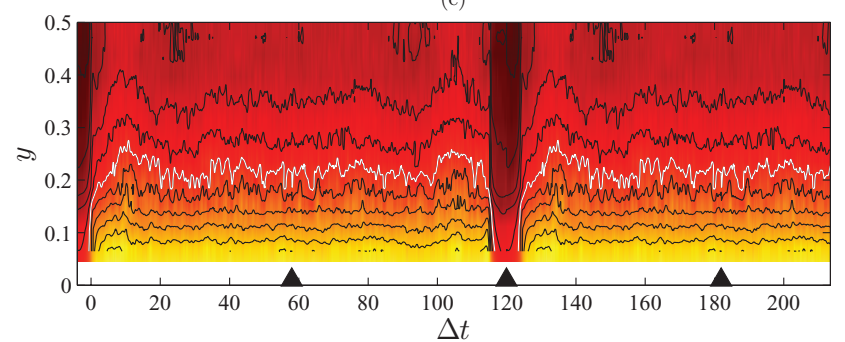

FIG. 19. Space-time distributions of conditionally sampled results at $R e=2290$. Representation is the same as in Figure 18 .

In the high $R e$ case shown in Figure 19, an up-and-down variation of $R e_{s}$ is observed in the turbulent part, in contrast to the monotonous increase of $R e_{s}$ at $R e=1650$. This undulation has a $30 d^{*} / U_{b}^{*}$ period and induces a higher-frequency component than that of the patch appearance. It is inferred that the departures of the very-low-frequency peak in the spectra maps (Figures 13 and 14) from the rate of the patch appearance in high-transitional $R e$ can be attributed to this undulation.

Figure 20 shows $R e$ dependence on these inclinations at the clipping time in the laminar and turbulent parts, $S=\frac{1}{R e} \frac{d R e_{s}}{d t}$. It is confirmed that the difference between the estimates from the switching conditions, LT or TL, is negligible. If the inclination is simply in proportion to the local $R e$ difference, $\Delta R e_{s}$, between the upstream and downstream interfaces, and in inverse proportion to the duration of the pure flow state, the slopes $S_{L}$ or $S_{T}$ would be represented by the following curves:

$$
\begin{gathered}
S_{L}=-\Delta \operatorname{Re}_{s} \frac{N}{1-\gamma}, \\
S_{T}=\Delta \operatorname{Re}_{s} \frac{N}{\gamma} .
\end{gathered}
$$

The inclinations following the curves illustrate that the velocity variation within each flow state is simply connecting the fringe edge velocities. This is also supported by the fact that the adopted values of the $\Delta R e_{s}, 0.16$ and 0.18 , for the laminar and turbulent inclinations, respectively, are very close to the difference between the laminar extrapolated values of $R e_{s} / R e$ to the interface time in Figures 17(a) and 17(b). We inferred that the discrepancy in the turbulence inclination at low $R e$ is from the inconstant difference of the extrapolated values, in contrast to those for the laminar state. The reason for disagreement regarding the turbulent inclination with the curve in the higher $R e$ is that there exists a turbulent part undulation, as described above. 


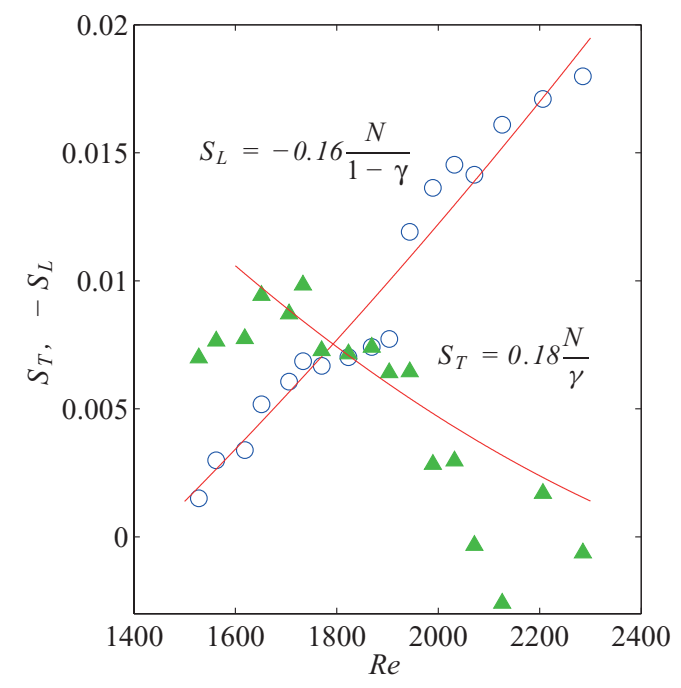

FIG. 20. Inclination of $\operatorname{Re}_{s}$ at the clipping time. Circles, laminar part; triangles, turbulent part.

\section{DISCUSSION AND CONCLUSION}

The streamwise velocity averaged in the wall-normal direction in the reconstructed flow maps indicates the large-scale temporal variation. Considering that turbulent patches in the visualized transitional flow ${ }^{10,11}$ are slowly deforming with longer temporal scale than their passing time, this temporal variation can be regarded as spatial change in the streamwise direction. As the velocity components averaged with respect to the wall-normal direction obey the two-dimensional continuous equation, their streamwise variation is concurrent with their spanwise variation. This indicates that there exist large flow structures in both the laminar and turbulent parts. The large-scale fluctuation is also confirmed in the velocity spectra maps as the peak at the very low frequency, and its frequency corresponds to an interval of the turbulent patch passing at the transitional $R e$. Around the upper marginal $R e$ the peak frequency still increases with increasing $R e$, departing from the rate of turbulent patch appearance. It appears that the departure at high intermittency is due to the undulation observed in the turbulent part in Figure 19.

An explanation for the large-scale flow structure forming is illustrated in Figure 21(a). As seen in Figure 18(b), the ensemble-averaged velocity, $\tilde{U}$ near the wall increases in the turbulent patch, indicating high skin friction there. This seems to be due to high momentum transfer in the wallnormal direction. The high skin friction slows the patch down ("A" in the illustration). We infer that the decelerating patch hits an upstream laminar flow producing a high-pressure region (B) that makes the upstream laminar flow slow down. Because of continuity of the flow, this decelerating flow turns to the spanwise direction resulting slanted spanwise streams (C) around the downstream interface of the turbulent patch. Similar two-dimensional flow around a turbulent band was observed

(a)

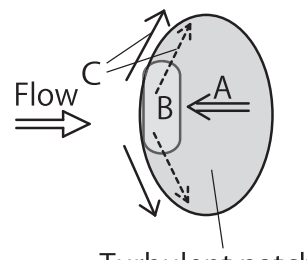

Turbulent patch (b)

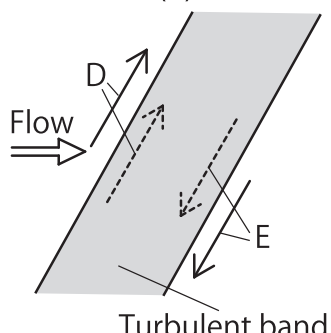

FIG. 21. Illustration of intermittent channel flows. (a) Turbulent patch. (b) Turbulent band. 
in the direct numerical simulation, ${ }^{12}$ and it is illustrated in Figure 21(b). Slanted streams around the downstream interface of the turbulent band (D) and counter streams around the upstream interface (E) appear after subtracting the bulk velocity. We infer that the counter streams result keeping overall flow rate to be zero in both streamwise and spanwise directions.

The scenario illustrated in Figure 21(a) well explains the peak at the center of the channel in the wall-normal distribution of the low-pass-filtered fluctuation around $R e=1500$, as seen in Figure 16. If a turbulent patch plays a role in a blockage, the pressure gradient in the streamwise direction uniformly acts over the whole channel width. Because of a viscous effect near the wall, the resultant velocity change is higher around the channel center. Therefore, the peak of the lowfrequency fluctuation appears at the channel center. The moderate-frequency velocity fluctuation, whose scale is about ten times as large as the channel width, is also observed in the premultiplied spectra near the wall. It is obvious that this scale is larger than the scale of the turbulent disturbance and smaller than the scale for the patch passing. Considering the streamwise scale, the moderate-scale fluctuation seems to be caused by longitudinal vortical structures that were investigated upstream and downstream of a turbulent patch in the flow visualization. ${ }^{10,11}$ The near-wall peak in the wall-normal distribution of the band-pass fluctuation also suggests that this moderate-scale fluctuation is due to the vortical disturbance.

The large flow structures induce curvature of the streamline in the laminar part, due to the local pressure gradient in the streamwise and spanwise directions. We conjecture that generation mechanism of the longitudinal vortical structures is similar to instability in the three-dimensional boundary layer developing on a swept wing. The stream curvature due to the blockage of the turbulent patch makes the local shear flow unstable because of unbalance between the pressure gradient and the centrifugal force, and then this instability induces the longitudinal vortical structures. No matter what generates the moderate-scale structures, the enhancement of momentum transfer in the wall-normal direction makes the laminar part profile turbulent-like, and then the profile is very similar to the turbulent part profile. The existence of the vortical structure is also supported by the fact that the shape factor drop is simultaneous with the increase of the band-pass-filtered fluctuation.

This momentum enhancement, however, leads to a contradiction that the deceleration of the laminar parts weakens the blockage effect of turbulent patches, which induces the pressure gradient that skews streamlines around the turbulent patches. The reduction of the blockage effect is insignificant in flows of very low intermittency around $R e=1500$, because the momentum enhancement in the laminar part is restricted in the vicinity of turbulent patches, and then fresh laminar flow hits the turbulent patches, one after another. As a result, the low-frequency fluctuation caused by the local pressure gradient appears at the channel center, as seen in the low-pass-filtered map in Figure 16. The fact that the laminar part profile at $R e=1520$ differs from that of the turbulent part, as shown in Figure 10, indicates that the laminar part is not filled up with disturbances inducing the wall-normal transfer of the momentum. With a decrease in the amount of the laminar parts with increasing $R e$, it becomes easy for the laminar part to be filled with the vortical structures. As seen in Figure 16, the band-pass-filtered fluctuation, which seems to be associable with the vortical structures, is predominant in the highly intermittent flow, $1600<R e<2000$. It is possible that the generation of the vortical structures by the streamwise skewness is delicately balanced with the blockage effect. This balance mechanism is also suggested by the fluctuation decline of the laminar part following the rapid increase up to $R e=1500$. The fluctuation due to the blockage effect increases with increasing intermittency factor as far as the vortical structure spreads into all of the laminar parts. In high intermittency factor cases, the vortical structure reduces the blockage effect, and then it is slightly weakened adversely.

In conclusion, we propose definitions of two marginal Reynolds numbers of channel flow. The upper marginal $R e$ is defined as the lowest $R e$ for fully turbulent channel flow. The lower marginal $R e$ is defined as the lowest $R e$ for intermittent channel flow with laminar and turbulent parts. Since the large-scale flow structure enables local regions of higher $R e$, turbulence can partly generate or survive even when the bulk $R e$ is lower than the limit for a sustainable turbulent flow, i.e., the upper marginal $R e$. The results of the streamwise energy decay and the intermittency factor dependence on the bulk $R e$ indicate that the lower marginal $R e$ is around 1400, though we observed turbulent 
patches lower than this value. For more precise determination of the lower marginal $R e$, a longer channel facility than the present one is needed.

As the spectral and conditional-average analyses indicated, the transitional channel flow has the large-scale flow structure with the turbulent patch appearance. To apply the concept, "edge state," to the transitional channel flow, the analysis should take this scale into account. The fact that the large-scale structures are observable even in the conditionally sampled flow field suggests that they are robust and long-lasting. If some flow structure appears frequently in the transitional channel flow, it is of great possibility that the edge-state captures its feature.

In the center spectra map of Figure 13, the peak of the large-scale disturbance continues from the transitional $R e$ to the turbulent flow at $R e=2660$ with little change of the peak frequency. In addition, the undulation of this scale is confirmed within the turbulent part just below the upper marginal $R e$. These results strongly suggest that the physical mechanism organizing the large-scale disturbance in the turbulent flow is the same in the transitional flow. The streamwise length scale of the disturbance equaling about 25 channel widths is very close to the superstructure in the fully developed turbulent channel ${ }^{33}$ at about $R e=140000$. The relation between them is one of the interesting open questions about turbulent channel flow.

\section{ACKNOWLEDGMENTS}

We thank Mr. Takeshi Okumura and Mr. Takayuki Numano for arranging the experimental setup and conducting pre-experiments. We also thank Mr. Yoshihiro Iwatani for his assistance with a part of the hot-wire measurements.

${ }^{1}$ S. A. Orszag, "Accurate solution of the Orr-Sommerfeld stability equation," J. Fluid Mech. 50, 689-703 (1971).

${ }^{2}$ S. J. Davies and C. M. White, "An experimental study of the flow of water in pipes of rectangular section," Proc. R. Soc. London, Ser. A 119, 92-107 (1928).

${ }^{3}$ V. C. Patel and M. R. Head, "Some observations on skin friction and velocity profiles in fully developed pipe and channel flows," J. Fluid Mech. 38, 181-201 (1969).

${ }^{4}$ M. A. Badri Narayanan, “An experimental study of reverse transition in two-dimensional channel flow," J. Fluid Mech. 31, 609-623 (1968).

${ }^{5}$ M. Nishioka and M. Asai, "Some observations of the subcritical transition in plane Poiseuille flow," J. Fluid Mech. 150, 441-450 (1985).

${ }^{6}$ D. R. Carlson, S. E. Widnall, and M. F. Peeters, “A flow visualization study of transition in plane Poiseuille flow," J. Fluid Mech. 121, 487-505 (1982).

${ }^{7}$ F. Alavyoon, D. S. Henningsson, and P. H. Alfredsson, "Turbulent spots in plane Poiseuille flow-flow visualization," Phys. Fluids 29, 1328-1331 (1982).

${ }^{8}$ S. A. Orszag and K. C. Kells, "Transition to turbulence in plane Poiseuille and plane Couette flow," J. Fluid Mech. 96, 159-205 (1980).

${ }^{9}$ O. Iida and Y. Nagano, "The relaminarization mechanisms of turbulent channel flow at low Reynolds numbers," Flow, Turbul. Combust. 60, 193-213 (1998).

${ }^{10}$ T. Okumura and M. Matsubara, "Experimental study on minimal Reynolds number with LDV measurement and visualization," in Proceedings of the 2006 Annual Meeting (Japan Society of Fluid Mechanics, 2006), p. 42.

${ }^{11}$ T. Natori, K. Watanabe, D. Aoki, D. Seki, and M. Matsubara, "Flow visualization in a relaminarzing channel flow," in Proceedings of the 2011 Annual Meeting (Japan Society of Fluid Mechanics, Hokuriku-Shinetsu Branch, 2011), pp. 75-76.

${ }^{12}$ K. Fukudome, O. Iida, and Y. Nagano, "Large-scale structure and the sustenance mechanisim in turbulent Poiseuille flow at low Reynolds number,” Trans. JSME B 76, 92-107 (2010).

${ }^{13}$ T. Tsukahara, Y. Kawaguchi, H. Kawamura, N. Tillmark, and P. H. Alfredsson, "Turbulence stripe in transitional channel flow with/without system rotation," in Proceedings of the Seventh IUTAM Symposium on Laminar-Turbulent Transition, IUTAM Bookseries Vol. 18 (Springer, 2010), pp. 421-426.

${ }^{14}$ J. D. Skufcaand, J. A. Yorke, and B. Eckhardt, "Edge of chaos in a parallel shear flow," Phys. Lett. Rev. 96, 174101 (2006).

${ }^{15}$ T. M. Schneider, B. Eckhardt, and J. A. Yorke, "Turbulence transition and the edge of chaos in pipe flow," Phys. Lett. Rev. 99, 034502 (2007).

${ }^{16}$ T. M. Schneider, J. F. Gibson, M. Lagha, F. D. Lillo, and B. Eckhardt, "Laminar-turbulent boundary in plane Couette flow," Phys. Rev. E 78, 037301 (2008).

${ }^{17}$ P. S. Y. Duguet and D. S. Henningson, "Localized edge state in plane Couette flow," Phys. Fluids 21, 111701 (2009).

${ }^{18}$ T. M. Schneider, J. F. Gibson, and J. Burke, "Snakes and ladders: Localized solution of plane Couette flow," Phys. Lett. Rev. 104, 104501 (2010).

${ }^{19}$ S. Cherubini, P. D. Palma, J. C. Robinet, and A. Bottaro, "Edge state in a boundary layer," Phys. Fluids 23, 051705 (2011).

${ }^{20}$ F. Mellibovsky, A. Meseguer, T. M. Schneider, and B. Eckhardt, “Transition in localized pipe flow turbulence,” Phys. Lett. Rev. 103, 054502 (2009). 
${ }^{21}$ Y. Duguet, A. P. Willis, and R. R. Kerswell, "Transition in pipe flow: The saddle structure on the boundary of turbulence," J. Fluid Mech. 613, 255-274 (2008).

${ }^{22}$ Y. Duguet, A. P. Willis, and R. R. Kerswell, "Slug genesis in cylindrical pipe flow," J. Fluid Mech. 663, 180-208 (2010).

${ }^{23}$ M. Asai and M. Nishioka, "Boundary-layer transition triggered by hairpin eddies at subcritical Reynolds numbers," J. Fluid Mech. 297, 101-122 (1995).

${ }^{24} \mathrm{P}$. H. Alfredsson and M. Matsubara, "Free-stream turbulence, streaky structure and transition in boundary layer flows," AIAA Paper No. 2000-2534, 2000.

${ }^{25}$ A. V. Johansson and P. H. Alfredsson, "On the structure of turbulent channel flow," J. Fluid Mech. 122, 295-314 (1982).

${ }^{26}$ B. G. B. Klingmann, "On transition due to three-dimensional disturbances in plane Poiseuille flow," J. Fluid Mech. 240, 167-195 (1992).

${ }^{27} \mathrm{P}$. T. Tsilingiris, "Thermophysical and transport properties of humid air at temperature range between 0 and $100{ }^{\circ} \mathrm{C}$," Energy Convers. Manage. 49, 1098-1110 (2008).

${ }^{28}$ T. B. Hedley and J. F. Keffer, "Turbulent/non-turbulent decisions in an intermittent flow," J. Fluid Mech. 64, 625-644 (1974).

${ }^{29}$ C. L. Kuan and T. Wang, "Investigation of the intermittent behavior of transitional boundary layer using a conditional averaging technique," Exp. Therm. Fluid Sci. 3, 157-173 (1990).

${ }^{30}$ J. Kim, P. Moin, and R. Moser, "Turbulence statistics in fully developed channel flow," J. Fluid Mech. 177, 133-166 (1987).

${ }^{31}$ M. Matsubara and P. H. Alfredsson, "Disturbance growth boundary layers in subjected to free stream turbulence," J. Fluid Mech. 430, 149-168 (2001).

${ }^{32}$ B. G. B. Klingmann and P. H. Alfredsson, "Turbulent spots in plane Poiseuille flow-Measurements of the velocity field," Phys. Fluids A 2, 2183-2195 (1990).

${ }^{33}$ J. P. Monty, J. A. Stewart, R. C. Williams, and M. S. Chong, "Large-scale features in turbulent pipe and channel flows," J. Fluid Mech. 589, 147-156 (2007). 